\title{
High-resolution global grids of revised Priestley-Taylor and Hargreaves-Samani coefficients for assessing ASCE-standardized reference crop evapotranspiration and solar radiation
}

\author{
Vassilis G. Aschonitis ${ }^{1}$, Dimitris Papamichail ${ }^{2}$, Kleoniki Demertzi ${ }^{2}$, Nicolo Colombani ${ }^{1}$, \\ Micol Mastrocicco $^{3}$, Andrea Ghirardini ${ }^{1}$, Giuseppe Castaldelli ${ }^{1}$, and Elisa-Anna Fano ${ }^{1}$ \\ ${ }^{1}$ Department of Life Sciences and Biotechnology, University of Ferrara, Ferrara, Italy \\ ${ }^{2}$ Department of Hydraulics, Soil Science and Agricultural Engineering, Aristotle University of Thessaloniki, \\ Thessaloniki, Greece \\ ${ }^{3}$ Department of Environmental, Biological and Pharmaceutical Sciences and Technologies, \\ University of Campania Luigi Vanvitelli, Caserta, Italy \\ Correspondence to: Vassilis G. Aschonitis (schvls@unife.it)
}

Received: 25 November 2016 - Discussion started: 23 December 2016

Revised: 21 June 2017 - Accepted: 18 July 2017 - Published: 25 August 2017

\begin{abstract}
The objective of the study is to provide global grids $\left(0.5^{\circ}\right)$ of revised annual coefficients for the Priestley-Taylor (P-T) and Hargreaves-Samani (H-S) evapotranspiration methods after calibration based on the ASCE (American Society of Civil Engineers)-standardized Penman-Monteith method (the ASCE method includes two reference crops: short-clipped grass and tall alfalfa). The analysis also includes the development of a global grid of revised annual coefficients for solar radiation $\left(R_{\mathrm{S}}\right)$ estimations using the respective $R_{\mathrm{S}}$ formula of $\mathrm{H}-\mathrm{S}$. The analysis was based on global gridded climatic data of the period 1950-2000. The method for deriving annual coefficients of the P-T and H-S methods was based on partial weighted averages (PWAs) of their mean monthly values. This method estimates the annual values considering the amplitude of the parameter under investigation $\left(\mathrm{ET}_{\mathrm{o}}\right.$ and $R_{\mathrm{S}}$ ) giving more weight to the monthly coefficients of the months with higher $\mathrm{ET}_{\mathrm{o}}$ values (or $R_{\mathrm{S}}$ values for the case of the H-S radiation formula). The method also eliminates the effect of unreasonably high or low monthly coefficients that may occur during periods where $\mathrm{ET}_{\mathrm{o}}$ and $R_{\mathrm{S}}$ fall below a specific threshold. The new coefficients were validated based on data from 140 stations located in various climatic zones of the USA and Australia with expanded observations up to 2016. The validation procedure for $\mathrm{ET}_{\mathrm{o}}$ estimations of the short reference crop showed that the P-T and H-S methods with the new revised coefficients outperformed the standard methods reducing the estimated root mean square error (RMSE) in $\mathrm{ET}_{\mathrm{o}}$ values by 40 and $25 \%$, respectively. The estimations of $R_{\mathrm{S}}$ using the H-S formula with revised coefficients reduced the RMSE by $28 \%$ in comparison to the standard H-S formula. Finally, a raster database was built consisting of (a) global maps for the mean monthly $\mathrm{ET}_{\mathrm{o}}$ values estimated by ASCE-standardized method for both reference crops, (b) global maps for the revised annual coefficients of the P-T and H-S evapotranspiration methods for both reference crops and a global map for the revised annual coefficient of the H-S radiation formula and (c) global maps that indicate the optimum locations for using the standard P-T and H-S methods and their possible annual errors based on reference values. The database can support estimations of $\mathrm{ET}_{\mathrm{o}}$ and solar radiation for locations where climatic data are limited and it can support studies which require such estimations on larger scales (e.g. country, continent, world). The datasets produced in this study are archived in the PANGAEA database (https://doi.org/10.1594/PANGAEA.868808) and in the ESRN database (http://www.esrn-database.org or http://esrn-database.weebly.com).
\end{abstract}




\section{Introduction}

The reference crop evapotranspiration $\mathrm{ET}_{\mathrm{O}}$ is defined as the maximum value of water losses by evaporation and transpiration above a reference crop (e.g. grass), which can be achieved under no water restrictions. It is also one of the most important parameters for water balance estimations and irrigation planning of crops (Allen et al., 1998). Several methods have been proposed for $\mathrm{ET}_{\mathrm{o}}$ estimations (Itenfisu et al., 2003; Allen et al., 2005; Wang and Dickinson, 2012; McMahon et al., 2013; Valipour, 2017; Valipour and Gholami Sefidkouhi, 2017; Valipour et al., 2017) with the most popular being the FAO-56 Penman-Monteith (Allen et al., 1998), the Priestley-Taylor (P-T; Priestley and Taylor, 1972) and the Hargreaves-Samani (Hargreaves and Samani, 1982, 1985) methods. The FAO-56 has been updated to the ASCE (American Society of Civil Engineers)-standardized method (Allen et al., 2005), which reflects the current state of the art, providing $\mathrm{ET}_{\mathrm{o}}$ estimations for two reference crops (a short and a tall reference crop, which correspond to clipped grass and alfalfa, respectively). The ASCE-standardized method has been proposed by the ASCE-EWRI (American Society of Civil Engineers - Environmental \& Water Resources Institute) Task Committee as the most precise method and requires a wide range of climatic parameters, which in many cases are not available. The problem of data availability can be solved by other methods, such as the Priestley-Taylor and HargreavesSamani, which require less information for their determination. In fact, they are considered the most precise among the simplified methods with reduced parameters (Xu and Singh, 2002; Sumner and Jacobs, 2005; Valipour, 2012, 2014).

The Priestley-Taylor method requires net solar radiation and temperature data. The P-T formula includes an empirical factor known as advection coefficient $a_{\mathrm{pt}}$, which is usually set equal to 1.26 (Priestley and Taylor, 1972) and generally ranges between 1.08 and 1.34 (Tateishi and Ahn, 1996; $\mathrm{Xu}$ et al., 2013). Other studies for various climatic conditions have shown that $a_{\mathrm{pt}}$ presents significant spatial and seasonal variability (Castellvi et al., 2001; Moges et al., 2003; Pereira, 2004; Tabari and Talaee, 2011; Aschonitis et al., 2015). Weiß and Menzel (2008) used the value 1.26 for wet and the value 1.75 for dry climatic conditions, as suggested by Maidment (1992). The value $a_{\mathrm{pt}}=1.26$ has been verified experimentally for bare irrigated soil (Eichinger et al., 1996). Theoretical simulations for the case of the reference crop in saturated soil have also verified $a_{\mathrm{pt}}=1.26$ for the case of no or restricted advection effects (Lhomme, 1997; McMahon et al., 2013). Lower values of the advection coefficient have been reported by Singh and Irmak (2011) $\left(a_{\mathrm{pt}}=1.14\right)$ for Nebraska (USA), by Abtew (1996) $\left(a_{\mathrm{pt}}=1.18\right)$ for Florida (USA), by Kellner (2001) $\left(a_{\mathrm{pt}}=0.8\right)$ for central Sweden, and by $\mathrm{Xu}$ and Singh (2002) $\left(a_{\mathrm{pt}}=0.9\right)$ for Switzerland. Values of $a_{\mathrm{pt}}<1$ have been reported for forested steep areas
(Shuttleworth and Calder, 1979; Giles et al., 1984; Flint and Childs, 1991). On the other hand, high values ranging between 1.82 and 2.14 have been reported for cold dry areas of Iran (Tabari and Talaee, 2011). Aschonitis et al. (2015) analysed the monthly variation in $a_{\mathrm{pt}}$ for Italian regions and observed through regression analysis that more than $90 \%$ of the spatial variability in the seasonal $a_{\mathrm{pt}}$ was explained by the spatial variability in vapour pressure deficit (DE; positive correlation). The rate of $a_{\mathrm{pt}}$ variation per unit DE was found significantly different between seasons, and it was negatively correlated to net solar radiation and/or temperature. The general trends in $a_{\mathrm{pt}}$ led to the conclusion that colder drier conditions due to low net radiation and high vapour pressure deficit tend to increase its values.

The Hargreaves-Samani (H-S) method requires only temperature data, including four empirical factors (or three depending on the formula). A part of the equation empirically describes the incident solar radiation $R_{\mathrm{s}}$. A basic problem of the Hargreaves-Samani method is that it tends to underestimate $\mathrm{ET}_{\mathrm{o}}$ under high-wind conditions $\left(u_{2}>3 \mathrm{~m} \mathrm{~s}^{-1}\right)$ and to overestimate $\mathrm{ET}_{\mathrm{o}}$ under conditions of high relative humidity (Allen et al., 1998). In the last years, many scientists have performed analysis and recalibration of the Hargreaves-Samani method for various climates (Trajkovic, 2007; Tabari, 2010; Tabari and Talaee, 2011; Azhar and Perera, 2011; Aschonitis et al., 2012; Mohawesh and Talozi, 2012; Rahimikhoob et al., 2012; Ravazzani et al., 2012; Bachour et al., 2013; Long et al., 2013; Mendicino and Senatore, 2013; Ngongondo et al., 2013; Berti et al., 2014; Heydari and Heydari, 2014), which indicates a global interest in simplified methods, mainly driven by the lack of data.

The analysis of $\mathrm{ET}_{\mathrm{o}}$ on a global scale is of special interest since it provides a general view of the spatiotemporal variation in this parameter, while (together with rainfall) it provides significant information about the aridity of terrestrial systems. A basic limitation of global analysis is the lack of homogeneously distributed meteorological stations around the globe and especially in mountainous regions. In the last years, climatic models, advanced interpolation and other methods have succeeded in generating datasets of various climatic parameters (Hijmans et al., 2005; Sheffield et al., 2006; Osborn and Jones, 2014; Brinckmann et al., 2016), facilitating the attempts to develop $\mathrm{ET}_{\mathrm{o}}$ maps. Significant work relating to global $\mathrm{ET}_{\mathrm{o}}$ estimations has been carried out by various scientists. Mintz and Walker (1993) used the Thorthwaite (1948) method and provided isoline maps of $\mathrm{ET}_{\mathrm{o}}$. Tateishi and Ahn (1996) used the PriestleyTaylor method and provided $\mathrm{ET}_{\mathrm{O}}$ maps at $0.5^{\circ}$ resolution. Droogers and Allen (2002) used the FAO-56 PenmanMonteith method, providing $\mathrm{ET}_{\mathrm{o}}$ maps at 10 arcmin resolution and a modified Hargreaves-Samani method, which considers rainfall. Weiß and Menzel (2008) compared four different methods (Priestley-Taylor, Kimberly Penman, FAO- 
56 Penman-Monteith and Hargreaves-Samani) and provided $\mathrm{ET}_{\mathrm{o}}$ maps at $0.5^{\circ}$ resolution. Zomer et al. (2008) used Hargreaves-Samani method and provided the highest resolution (30 arcsec) available for $\mathrm{ET}_{\mathrm{O}}$ maps.

The objectives of the study are

a. to develop mean monthly maps of $\mathrm{ET}_{\mathrm{o}}$ for the period 1950-2000 on a global scale using the most precise ASCE-standardized method for both reference crops (short-clipped grass and tall alfalfa)

b. to develop global maps that provide the possible annual error in $\mathrm{ET}_{\mathrm{o}}$ estimations using the standard P-T and H-S evapotranspiration methods in comparison to the ASCE method for the short reference crop and the possible annual error in solar radiation estimations using the temperature-based $\mathrm{H}-\mathrm{S}$ radiation formula (this attempt will allow us to identify the optimum locations for the application of the standard H-S and P-T evapotranspiration formulas based on their proximity to the results of ASCE for the short reference crop)

c. to develop global maps of readjusted annual coefficients for the H-S and P-T evapotranspiration methods for both short and tall reference crop based on a new method that estimates partial weighted averages of the monthly coefficients (the same procedure was also followed for the coefficients of the $\mathrm{H}-\mathrm{S}$ radiation formula)

d. to validate the results of the readjusted P-T and $\mathrm{H}$ $\mathrm{S}$ coefficients using data from meteorological stations from different locations with different climatic conditions and

e. to compare the predictive ability of the readjusted P-T and $\mathrm{H}-\mathrm{S}$ coefficients for the short reference crop evapotranspiration with the respective predictions obtained from other models that have low data requirements.

The analysis and the produced datasets of this study were based on mean monthly climatic data of $0.5^{\circ}$ resolution for the period 1950-2000. The final datasets of revised H-S and P-T coefficients will provide a global overview of the variation in their values and a common base for comparing the values of different regions since they are calibrated using common datasets and using the same technique. The produced global datasets of this study can support estimations of $\mathrm{ET}_{\mathrm{o}}$ and solar radiation for locations where climatic data are limited, and it can support studies which require such estimations on larger scales (e.g. country, continent, world).

\section{Data and methods}

\subsection{Global climatic data}

The analysis presented in this study was based on global climatic data obtained from the following databases:
- The database of Hijmans et al. (2005) provides mean monthly values for the parameters of precipitation and maximum, minimum and mean temperature at 30 arcsec spatial resolution. The data are provided as grids of mean monthly values of the period 1950-2000 (http: //www.worldclim.org/). The database also includes a revised digital elevation model (DEM) of GTOPO30 (global topographic at 30 arcsec) based on SRTM (Shuttle Radar Topographic Mission), which was used for the estimation of atmospheric pressure. The DEM was also used as a base to calculate the distance from the coastlines in raster format at 30 arcsec spatial resolution based on the Euclidean distance.

- The database of Sheffield et al. (2006) provides monthly values of parameters such as wind speed at the height of $10 \mathrm{~m}$ above the ground surface, solar radiation, specific humidity, precipitation and temperature for the period $1948-2006$ at $0.5^{\circ}$ spatial resolution. The data are available in the form of netcdf files of monthly values of each year for the period 1948-2006 (http://hydrology. princeton.edu/data.pgf.php).

- The database of Peel et al. (2007) provides the revised global Köppen-Geiger climate map. The data are provided in raster form with 0.1 degree spatial resolution. The climate map was developed using the GHCN (Global Historical Climatology Network) version 2.0 dataset (Peterson and Vose, 1997), which includes precipitation data from 12396 stations and temperature data from 4844 station data for the periods 1909-1991 and 1923-1993, respectively. The Köppen-Geiger map was used to obtain the climatic type of the meteorological stations used in the validation dataset.

In this study, the $\mathrm{ET}_{\mathrm{o}}$ is estimated combining the databases of Hijmans et al. (2005) and Sheffield et al. (2006), as follows: (a) mean monthly values of maximum, minimum and mean temperature and precipitation were obtained from Hijmans et al. (2005), while (b) wind speed, specific humidity and incident solar radiation were obtained from the Sheffield et al. (2006) database. The specific humidity was converted to actual vapour pressure using the equation given by Peixoto and Oort (1996). The final results and analysis presented in this study are based on the coarser $0.5^{\circ}$ resolution.

All the calculations presented in the next sections were performed in an ArcGIS 9.3 ESRI environment in a WGS84 ellipsoid coordinate system. For area coverage calculations or for estimations of mean global values of various parameters, coordinate system conversions were performed from WGS84 to projected Cylindrical Equal Area system (any percentage global coverage calculations and the derivation of mean global values of various parameters are performed without considering Antarctica). 


\subsection{Methods}

\subsubsection{The ASCE standardized reference evapotranspiration method}

The estimation of $\mathrm{ET}_{\mathrm{O}}$ using the ASCE method is performed by the following equation (Allen et al., 2005):

$\mathrm{ET}_{\mathrm{o}}=\frac{0.408 \Delta\left(R_{\mathrm{n}}-G\right)+\frac{\gamma u_{2}\left(e_{\mathrm{s}}-e_{\mathrm{a}}\right) C_{\mathrm{n}}}{\left(T_{\text {mean }}+273.16\right)}}{\Delta+\gamma\left(1+C_{\mathrm{d}} u_{2}\right)}$,

where $\mathrm{ET}_{\mathrm{o}}$ is the reference crop evapotranspiration $\left(\mathrm{mm} \mathrm{d}^{-1}\right), R_{\mathrm{n}}$ is the net solar radiation at the crop surface $\left(\mathrm{MJ} \mathrm{m}^{-2} \mathrm{~d}^{-1}\right), u_{2}$ is the wind speed at $2 \mathrm{~m}$ height above the soil surface $\left(\mathrm{m} \mathrm{s}^{-1}\right), T_{\text {mean }}$ is the mean daily air temperature $\left({ }^{\circ} \mathrm{C}\right), G$ is the soil heat flux density at the soil surface $\left(\mathrm{MJ} \mathrm{m}^{-2} \mathrm{~d}^{-1}\right), e_{\mathrm{S}}$ is the saturation vapour pressure $(\mathrm{kPa}), e_{\mathrm{a}}$ is the actual vapour pressure $(\mathrm{kPa}), \Delta$ is the slope of the saturation vapour pressure-temperature curve $\left(\mathrm{kPa}^{\circ} \mathrm{C}^{-1}\right), \gamma$ is the psychrometric constant $\left(\mathrm{kPa}^{\circ} \mathrm{C}^{-1}\right)$ and $C_{\mathrm{n}}$ and $C_{\mathrm{d}}$ are constants, which vary according to the time step and the reference crop type and describe the bulk surface resistance and aerodynamic roughness. The short reference crop (ASCEshort) corresponds to clipped grass of $12 \mathrm{~cm}$ height and surface resistance of $70 \mathrm{~s} \mathrm{~m}^{-1}$, where the constants $C_{\mathrm{n}}$ and $C_{\mathrm{d}}$ have the values 900 and 0.34 , respectively. The tall reference crop (ASCE-tall) corresponds to full-cover alfalfa of $50 \mathrm{~cm}$ height and surface resistance of $45 \mathrm{~s} \mathrm{~m}^{-1}$, where the constants $C_{\mathrm{n}}$ and $C_{\mathrm{d}}$ have the values 1600 and 0.38 , respectively (Allen et al., 2005). The use of Eq. (1) at a daily or monthly step for the short reference crop is equivalent to the FAO-56 method (Allen et al., 1998).

\subsubsection{The Priestley-Taylor method}

The calculation of Priestley-Taylor (P-T) method is performed by the following equation (Priestley and Taylor, 1972):

$\mathrm{ET}_{\mathrm{o}}=a_{\mathrm{pt}} \frac{\Delta}{\lambda(\Delta+\gamma)}\left(R_{\mathrm{n}}-G\right)$,

where $\mathrm{ET}_{\mathrm{o}}$ is the potential evapotranspiration $\left(\mathrm{mm} \mathrm{d}^{-1}\right), R_{\mathrm{n}}$ is the net solar radiation $\left(\mathrm{MJ} \mathrm{m}^{-2} \mathrm{~d}^{-1}\right), G$ is the soil heat flux density at the soil surface $\left(\mathrm{MJ} \mathrm{m}^{-2} \mathrm{~d}^{-1}\right), \Delta$ is the slope of the saturation vapour pressure-temperature curve $\left(\mathrm{kPa}^{\circ} \mathrm{C}^{-1}\right), \gamma$ is the psychrometric constant $\left(\mathrm{kPa}^{\circ} \mathrm{C}^{-1}\right), \lambda$ is the latent heat of vaporization ( $\mathrm{MJ} \mathrm{kg}^{-1}$ ) and $a_{\mathrm{pt}}$ is the P-T advection coefficient. The value of $\lambda$ was considered equal to $2.45 \mathrm{MJ} \mathrm{kg}^{-1}$ (Allen et al., 1998) (this value is also constant in Eq. (1) and appears as $1 / \lambda=0.408$ ). Equation (1) strictly refers to the reference crop evapotranspiration (i.e. short or tall crop), whereas Eq. (2) has been used for the calculation of evapotranspiration under non-limiting water conditions of the short reference crop, bare soil or open-water surface and for this reason is also called potential evapotranspiration, which is a more general term. Equation (2) is applied in this study as a reference crop evapotranspiration method and for this reason is compared with Eq. (1) for the short reference crop using the standard mean global value 1.26 for the factor $a_{\mathrm{pt}}$.

\subsubsection{The Hargreaves-Samani method}

The Hargreaves-Samani (H-S) method (Hargreaves and Samani, 1982, 1985) for $\mathrm{ET}_{\mathrm{o}}$ includes an internal function, which estimates the incoming shortwave solar radiation $R_{\mathrm{S}}$ $\left(\mathrm{MJ} \mathrm{m}^{-2} \mathrm{day}^{-1}\right)$ as follows:

$R_{\mathrm{S}}=K_{\mathrm{RS}} \cdot R_{\mathrm{a}} \cdot(\mathrm{TD})^{0.5}$,

where $K_{\mathrm{RS}}$ is the adjustment coefficient for the H-S radiation formula $\left({ }^{\circ} \mathrm{C}^{-0.5}\right), R_{\mathrm{a}}$ is the extraterrestrial radiation $\left(\mathrm{MJ} \mathrm{m}^{-2} \mathrm{day}^{-1}\right)$, and TD is the temperature difference between maximum and minimum daily temperature $\left({ }^{\circ} \mathrm{C}\right)$. According to Allen et al. (1998), the empirical $K_{\mathrm{RS}}$ coefficient differs for "interior" or "coastal" regions: (a) $K_{\mathrm{RS}}=0.16$ for interior locations, where land mass dominates and air masses are not strongly influenced by a large water body, and (b) $K_{\mathrm{RS}}=0.19$ for coastal locations, situated on or adjacent to the coast of a large land mass and where air masses are influenced by a nearby water body. For general use of Eq. (3), a mean global value of $K_{\mathrm{RS}}=0.17$ has been adopted in this study. $R_{\mathrm{a}}$ and $R_{\mathrm{S}}$ divided by $\lambda$ change units from $\mathrm{MJ} \mathrm{m}^{-2}$ day $^{-1}$ to $\mathrm{mm} \mathrm{day}^{-1}$ as it is required in the next equation of $\mathrm{ET}_{\mathrm{o}}$. The formula for estimating $\mathrm{ET}_{\mathrm{o}}$ by the $\mathrm{H}-\mathrm{S}$ method is given by the following equation (Hargreaves and Samani, 1982, 1985):

$$
\begin{aligned}
\mathrm{ET}_{\mathrm{o}} & =0.0135\left(T_{\text {mean }}+17.8\right) \frac{R_{\mathrm{s}}}{\lambda} \\
& =0.0135\left(T_{\text {mean }}+17.8\right) K_{\mathrm{RS}} \cdot \frac{R_{\mathrm{a}}}{\lambda} \cdot(\mathrm{TD})^{0.5}
\end{aligned}
$$

Considering Eq. (4a), the $K_{\mathrm{RS}}$ and the exponent 0.5 are adjustment factors of the radiation formula (Eq. 3), while 0.0135 and 17.8 are adjustment factors of the $\mathrm{ET}_{\mathrm{o}}$ formula, leading to a total amount of four empirical coefficients. Using the mean global value of $K_{\mathrm{RS}}=0.17$, Eq. (4a) is simplified according to the following (Allen et al., 1998):

$$
\begin{aligned}
\mathrm{ET}_{\mathrm{o}} & =c_{\mathrm{hs} 2} \cdot\left(T_{\text {mean }}+17.8\right) \cdot \frac{R_{\mathrm{a}}}{\lambda} \cdot(\mathrm{TD})^{0.5} \\
& =0.0023\left(T_{\text {mean }}+17.8\right) \cdot \frac{R_{\mathrm{a}}}{\lambda} \cdot(\mathrm{TD})^{0.5},
\end{aligned}
$$

where in both Eqs. (4a) and (4b), the $\mathrm{ET}_{\mathrm{o}}$ is the potential evapotranspiration $\left(\mathrm{mm} \mathrm{d}^{-1}\right), R_{\mathrm{a}}$ is the extraterrestrial radiation $\left(\mathrm{MJ} \mathrm{m}^{-2} \mathrm{~d}^{-1}\right), \lambda$ is the latent heat of vaporization $\left(\mathrm{MJ} \mathrm{kg}^{-1}\right)$ and $T_{\text {mean }}$ is the mean daily temperature $\left({ }^{\circ} \mathrm{C}\right)$. Eq. (4b) is applied in this study as a reference crop evapotranspiration method and for this reason is compared with Eq. (1) for the short reference crop. For the case of $T_{\text {mean }}<$ $-17.8^{\circ} \mathrm{C}$, the term of $\left(T_{\text {mean }}+17.8\right)$ was set to 0 , which is necessary for a global application (Weiß and Menzel, 2008). 
In further steps of analysis, the coefficient 0.0135 (Eq. 4a) is represented as $c_{\mathrm{hs} 1}$, while the coefficient 0.0023 is represented as $c_{\mathrm{hs} 2}$, which is equal to $c_{\mathrm{hs} 2}=c_{\mathrm{hs} 1} \cdot K_{\mathrm{RS}}$.

In order to reduce the errors of the aforementioned methods in the high latitudes and altitudes (polar and alpine environments) where negative temperatures exist, a filter was used in all methods to set mean monthly $\mathrm{ET}_{\mathrm{o}}=0$ when mean monthly $T_{\max }$ is $\leq 0$ (conditions of extreme frost).

\subsubsection{Steps of analysis}

Step 1: comparative analysis between the standard $\mathrm{ET}_{\mathrm{O}}$ formulas of ASCE, P-T and H-S and error analysis of $\mathrm{H}-\mathrm{S}$ radiation formula

The first step of the analysis includes the estimation of mean monthly and mean annual $\mathrm{ET}_{\mathrm{o}}$ using the ASCE method (Eq. 1) for the two reference crops (short and tall), the standard P-T method (Eq. 2) with $a_{\mathrm{pt}}=1.26$ and the standard $\mathrm{H}-\mathrm{S}$ method according to Eq. (4b) with $c_{\mathrm{hs} 2}=0.0023$. The difference between the $\mathrm{ET}_{\mathrm{o}}$ methods will be captured using as a base the mean annual and the mean monthly $\mathrm{ET}_{\mathrm{o}}$ values of ASCE-short.

In the case of mean annual $\mathrm{ET}_{\mathrm{o}}$, the analysis is based on the percentage of mean annual difference (MAD \%) of each method $M$ versus the mean annual $\mathrm{ET}_{\mathrm{o}}$ of ASCE-short, which is given by

$\operatorname{MAD}_{(M)}=100\left[\mathrm{YET}_{\mathrm{o}(M)}-\mathrm{YET}_{\mathrm{os}}\right] /\left(\mathrm{YET}_{\mathrm{os}}\right)$,

where $\mathrm{YET}_{\mathrm{os}}$ is the mean annual $\mathrm{ET}_{\mathrm{O}}$ of ASCE-short method, $\mathrm{YET}_{\mathrm{O}(M)}$ is the mean annual $\mathrm{ET}_{\mathrm{o}}$ of the $M$ method ( $M$ can be either the ASCE-tall method or the standard P-T method or the standard H-S method). The MAD\% for ASCE-tall was estimated in order to assess the effects of reference crop type at different climatic environments on the annual estimations of $\mathrm{ET}_{\mathrm{o}}$. The MAD \% of the P-T and H-S methods was used to investigate the strength of the two standard methods to approximate the annual $\mathrm{ET}_{\mathrm{o}}$ of ASCE-short. Positive values of MAD \% indicate overestimation of the mean annual $\mathrm{ET}_{\mathrm{O}}$ values using the $M$ method in comparison to the ASCE-short method, while negative values indicate underestimation. Furthermore, the difference between the absolute MAD \% values (DMAD) of the standard P-T (with $a_{\mathrm{pt}}=1.26$ ) and H-S (with $c_{\mathrm{hs} 2}=0.0023$ ) methods was estimated in order to assess which of the two methods is more appropriate to be used locally, based on its proximity to the ASCE-short method. The DMAD is estimated as follows:

$\mathrm{DMAD}=\left|\mathrm{MAD}_{(\mathrm{H}-\mathrm{S})}\right|-\left|\mathrm{MAD} \%_{(\mathrm{P}-\mathrm{T})}\right|$,

where positive DMAD values indicate better performance of standard P-T, while negative values indicate better performance of the standard H-S method in a region. Regions that showed DMAD values between -1 and +1 were considered transitional zones where both methods showed approximately the same proximity to the annual ASCE-short estimations.
In the case of mean monthly $\mathrm{ET}_{\mathrm{o}}$, the coefficient of determination $R^{2}$ and the root mean square difference (RMSD; equivalent to the root mean square error, RMSE) (Droogers and Allen, 2002) were used to compare the mean monthly values of ASCE-tall, standard P-T $\left(a_{\mathrm{pt}}=1.26\right)$ and standard $\mathrm{H}-\mathrm{S}\left(c_{\mathrm{hs} 2}=0.0023\right)$ methods with the respective values of the ASCE-short method.

The procedures of MAD \%, $R^{2}$ and RMSD were also used to analyse the mean annual and mean monthly estimations of $R_{\mathrm{S}}$ by the standard solar radiation formula of H-S (Eq. 3 with $\left.K_{\mathrm{RS}}=0.17\right)$ versus the given $R_{\mathrm{S}}$ values of Sheffield et al. (2006).

\section{Step 2: readjustment of annual P-T and H-S coefficients} for both reference crops

For the case of P-T, the readjustment of the mean monthly $a_{\mathrm{pt}}$ coefficient was performed directly for each location by solving for $a_{\mathrm{pt}}$ after equating Eq. (1) with Eq. (2) of each month. A filter was used in order to set $a_{\mathrm{pt}}$ equal to 0 when Eq. (1) and/or Eq. (2) without $a_{\mathrm{pt}}$ were equal to 0 . In this case, the $a_{\mathrm{pt}}$ changes its physical meaning in order to indicate that mean monthly $\mathrm{ET}_{\mathrm{o}}$ approximates to 0 . By carrying out the above procedures for both short and tall reference crop, 12 images of mean monthly readjusted $a_{\mathrm{pt}}$ coefficients were produced for each reference crop.

For the case of the H-S method, the readjustment of the coefficients was performed in two stages. In the first stage, the readjustment was performed in the radiation formula (Eq. 3) only for the $K_{\mathrm{RS}}$ coefficient, while the exponent 0.5 (square root) of the DT remained the same. The mean monthly $K_{\mathrm{RS}}$ was estimated using the values of solar radiation $R_{\mathrm{S}}$ given by Sheffield et al. (2006). In the second stage, the readjustment was performed in the evapotranspiration formula (Eq. 4b) for the coefficient of $c_{\mathrm{hs} 2}$ using as a base the ASCE method for both reference crops, while the coefficients of 17.8 and 0.5 remained the same. In this way the readjusted values of $c_{\mathrm{hs} 2}$ and $K_{\mathrm{RS}}$ would also provide readjusted values of the $c_{\mathrm{hs} 1}$ since $c_{\mathrm{hs} 1}=c_{\mathrm{hs} 2} / K_{\mathrm{RS}}$. A similar filter to set $c_{\mathrm{hs} 2}=0$ as in the case of $a_{\mathrm{pt}}$ was used, when Eq. (1) and/or Eq. (4b) without $c_{\mathrm{hs} 2}$ were equal to 0 . Following the above procedures, 12 images of mean monthly readjusted $c_{\mathrm{hs} 2}$ coefficients for each reference crop (short and tall) and $12 K_{\mathrm{RS}}$ images were produced.

The new mean monthly $a_{\mathrm{pt}}, c_{\mathrm{hs} 2}$ and $K_{\mathrm{RS}}$ coefficients were used to build respective mean annual coefficients. The robustness of mean annual coefficients are strongly related to their ability to capture the values of the dependent variable better (i.e. $\mathrm{ET}_{\mathrm{o}}$ and $R_{\mathrm{S}}$ ), especially in the months that present larger values. For this reason, weighted annual averages of mean monthly $a_{\mathrm{pt}}, c_{\mathrm{hs} 2}$ and $K_{\mathrm{RS}}$ coefficients were estimated. Under cold conditions, the monthly coefficients may present unrealistic values that significantly affect the weighted averages. In order to solve this problem, threshold values for the mean monthly dependent variables (i.e. $\mathrm{ET}_{\mathrm{o}}$ and $R_{\mathrm{S}}$ ) were set 
before their inclusion in the weighted average estimations. Preliminary analysis for the readjustment of $a_{\mathrm{pt}}$ and $c_{\mathrm{hs} 2}$ coefficients (based on the values of ASCE-short) showed that when the mean monthly ET $_{\mathrm{O}}$ values of ASCE-short, $\mathrm{H}-\mathrm{S}$ and P-T were below $45 \mathrm{~mm} \mathrm{month}^{-1}\left(\sim 1.5 \mathrm{~mm} \mathrm{~d}^{-1}\right)$, unrealistic mean monthly values of $a_{\mathrm{pt}}$ and $c_{\mathrm{hs} 2}$ coefficients appeared. The values considered unrealistic were those that were at least 1 order of magnitude larger or smaller than the standard values of $a_{\mathrm{pt}}=1.26$ and $c_{\mathrm{hs} 2}=0.0023$. Taking into account the above, the following procedure was performed in order to obtain partial weighted annual averages (after excluding months with $\mathrm{ET}_{\mathrm{o}} \leq 45 \mathrm{~mm} \mathrm{month}^{-1}$ ) of mean monthly $a_{\mathrm{pt}}$ and $c_{\mathrm{hs} 2}$ coefficients for the short reference crop based on the ASCE-short method:

when $\mathrm{ET}_{\mathrm{os} i}>45 \mathrm{mmmonth}^{-1}, \mathrm{Fr}_{i}=1$ else $=0$,

and

when $\mathrm{ET}_{\mathrm{oi}(M)}>45 \mathrm{~mm}_{\text {month }}{ }^{-1}, \mathrm{Fm}_{i}=1$ else $=0$.

$\mathrm{ET}_{\text {os adj. } i}=\mathrm{ET}_{\mathrm{os} \mathrm{i}} \cdot \mathrm{Fr}_{i} \cdot \mathrm{Fm}_{i}$

YET $_{\text {os adj. }}=\sum_{i=1}^{12}\left(\mathrm{ET}_{\text {os adj. } . i}\right)$

$C=\sum_{i=1}^{12}\left(\frac{\mathrm{ET}_{\text {os adj. } i}}{\mathrm{YET}_{\mathrm{os} \text { adj. }}} \cdot C_{i}\right)$,

where $\mathrm{ET}_{\mathrm{os} i}$ is the mean monthly value of $\mathrm{ET}_{\mathrm{O}}$ (mmmonth ${ }^{-1}$ ) obtained from the ASCE-short method, $\mathrm{ET}_{\mathrm{oi}(M)}$ is the mean monthly value of $\mathrm{ET}_{\mathrm{o}}\left(\mathrm{mm} \mathrm{month}^{-1}\right)$ obtained from the $M$ method ( $M$ is either P-T or H-S), $\mathrm{Fr}_{i}$ is the filter function of the reference method with values 0 or $1, \mathrm{Fm}_{i}$ is the filter function of the $M$ method with values 0 or $1, \mathrm{ET}_{\mathrm{os} \text { adj. } i}$ is the adjusted mean monthly value of $\mathrm{ET}_{\mathrm{O}}$ from the ASCE-short method, which becomes 0 when $\operatorname{Fr}_{i}$ or $\mathrm{Fm}_{i}$ is $0, \mathrm{YET}_{\mathrm{os} \text { adj. }}$ is the sum of the monthly adjusted

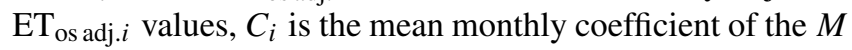
method (i.e. $a_{\mathrm{pt}}$ or $c_{\mathrm{hs} 2}$ ) calibrated based on the ASCE-short method (results from the previous step of the analysis), $C$ is the partial weighted average (PWA) of the mean monthly coefficients of the $M$ method (i.e. $a_{\mathrm{pt}}$ or $c_{\mathrm{hs} 2}$ ) for the short reference crop, and $i$ is month.

For estimating the PWA of mean monthly $a_{\mathrm{pt}}$ and $c_{\mathrm{hs} 2}$ for the tall reference crop, the same procedure as in Eq. (7) was followed using the mean monthly values of $\mathrm{ET}_{\mathrm{o}}$ from ASCEtall to estimate the $\mathrm{Fr}_{i}$ values in Eq. (7a), while the adjusted

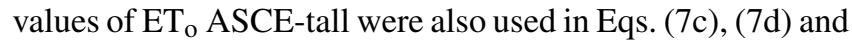
(7e). For $C_{i}$ values in Eq. (7e), the estimated mean monthly values of $a_{\mathrm{pt}}$ or $c_{\mathrm{hs} 2}$ based on the ASCE-tall method were used. Even though the mean monthly values of ASCE-tall are generally higher than those from ASCE-short, the threshold of $45 \mathrm{~mm} \mathrm{month}^{-1}$ in Eqs. (7a) and (7b) remained the same since it was observed that the difference between ASCEshort and ASCE-tall is very small when $\mathrm{ET}_{\mathrm{os} i}$ falls below $\sim 50 \mathrm{~mm} \mathrm{month}^{-1}$.
A similar procedure (using the set of Eqs. 7) was also followed to obtain the PWA of mean monthly $K_{\mathrm{RS}}$ of the $\mathrm{H}-\mathrm{S}$ method for $R_{\mathrm{S}}$ estimations. The $\mathrm{Fr}_{i}$ values in Eq. (7a) were estimated using as reference the mean monthly $R_{\mathrm{S}}$ values of Sheffield et al. (2006), which were also used after adjustment in Eqs. (7c)-(7e). The $\mathrm{Fm}_{i}$ values in Eq. (7b) were estimated using the respective $R_{\mathrm{S}}$ values of the standard H-S with $K_{\mathrm{RS}}=0.17$. For $C_{i}$ values in Eq. (7e), the mean monthly values of $K_{\mathrm{RS}}$ calibrated based on the $R_{\mathrm{S}}$ values of Sheffield et al. (2006) were used. The threshold used for adjusting $R_{\mathrm{S}}$ values in Eqs. (7a) and (7b) was set a equal to $3.61 \mathrm{MJ} \mathrm{m}^{-2} \mathrm{~d}^{-1}\left(\sim 110 \mathrm{MJ} \mathrm{m}^{2}\right.$ month $\left.^{-1}\right)$, which is equivalent to $45 \mathrm{~mm} \mathrm{month}^{-1}$ (conversion of $\mathrm{mm} \mathrm{month}^{-1}$ to $\mathrm{MJ} \mathrm{m}^{-2}$ month $^{-1}$ was performed after multiplying with $\lambda=$ $2.45 \mathrm{MJ} \mathrm{kg}^{-1}$ ). The threshold for $R_{\mathrm{s}}$ adjustment was tested before its use, and it was found to work satisfactorily excluding unrealistic monthly values of $K_{\mathrm{RS}}$. The values that were considered unrealistic were those that were at least 1 order of magnitude larger or smaller than the standard value of $K_{\mathrm{RS}}=0.17$.

\section{Step 3: use of stations for the validation of the PWA coefficients of the P-T and H-S methods and comparisons with other models of reduced parameters}

Stations from two databases (the California Irrigation Management System CIMIS database, http://www.cimis.water. ca.gov, and the Australian Government Bureau of Meteorology AGBM database, http://www.bom.gov.au/) were used in this study in order to validate the PWA coefficients of the $\mathrm{P}-\mathrm{T}$ and $\mathrm{H}-\mathrm{S}$ methods. The first database includes stations from California (USA), and it was selected for the following reasons: (a) it has been used as a basis for the development of the Hargreaves-Samani method (Hargreaves and Samani, 1985; Hargreaves and Allen, 2002) and the CIMIS method (Snyder and Pruitt, 1985, 1992), and (b) it provides a dense and descriptive network of stations for a specific region that combines coastal, plain, mountain and desert environments (Table 1, Fig. 1a). The second database includes stations from Australia, and it was selected because the stations network covers a large territory with a large variety of climate classes (Table 1, Fig. 1b) but also because the PriestleyTaylor method has been calibrated for locations of eastern Australia (Priestley and Taylor, 1972). The selection of stations from the AGBM database was performed in such way as to cover all the possible existing Köppen climatic types and elevation ranges of the Australian continent (Table 1). In total, 140 stations were used; 60 stations were selected from CIMIS and 80 stations from the AGBM that have at least 15 years of observations (some stations that do not follow this rule were selected due to their special climate Köppen class or the high elevation of their location). Observations from the years after 2000 up to 2016 were included (if they were available) in the station data, in order to show that the new revised coefficients are applicable to recent periods. 
Table 1. Meteorological stations from the USA (California; USA-CA) (CIMIS database) and Australia (AGBM database).

\begin{tabular}{|c|c|c|c|c|c|c|c|c|}
\hline No. & Code & Station & Country & $\begin{array}{r}\text { Elevation } \\
(\mathrm{m})\end{array}$ & $\begin{array}{r}\text { Lat } \\
\text { (Dec. deg.) }\end{array}$ & $\begin{array}{r}\text { Long } \\
\text { (Dec. deg.) }\end{array}$ & Period & $\begin{array}{l}\text { Köppen } \\
\text { class }^{\text {a }}\end{array}$ \\
\hline CA-1 & 006 & Davis & USA-CA & 18 & 38.54 & -121.78 & Sep 1982-Aug 2016 & Csa \\
\hline CA-2 & 002 & FivePoints & USA-CA & 87 & 36.34 & -120.11 & Jun 1982-Aug 2016 & BWk \\
\hline $\mathrm{CA}-3$ & 005 & Shafter & USA-CA & 110 & 35.53 & -119.28 & Jun 1982-Aug 2016 & BSk \\
\hline $\mathrm{CA}-4$ & 007 & Firebaugh/Telles & USA-CA & 56 & 36.85 & -120.59 & Sep 1982-Aug 2016 & BWk \\
\hline CA-5 & 012 & Durham & USA-CA & 40 & 39.61 & -121.82 & Oct 1982-Aug 2016 & Csa \\
\hline CA-6 & 008 & Gerber & USA-CA & 76 & 40.04 & -122.17 & Sep 1982-Aug 2014 & Csa \\
\hline $\mathrm{CA}-7$ & 015 & Stratford & USA-CA & 59 & 36.16 & -119.85 & Nov 1982-Aug 2016 & BSk \\
\hline CA-8 & 019 & Castroville & USA-CA & 3 & 36.77 & -121.77 & Nov 1982-Aug 2016 & $\mathrm{Csb}$ \\
\hline CA-9 & 021 & Kettleman & USA-CA & 104 & 35.87 & -119.89 & Nov 1982-Aug 2016 & BWk \\
\hline CA-10 & 027 & Zamora & USA-CA & 15 & 38.81 & -121.91 & Dec 1982-May 2006 & Csa \\
\hline CA-11 & 030 & Nicolaus & USA-CA & 10 & 38.87 & -121.55 & Jan 1983-Dec 2011 & Csa \\
\hline CA-12 & 032 & Colusa & USA-CA & 17 & 39.23 & -122.02 & Jan 1983-Aug 2016 & Csa \\
\hline CA-13 & 033 & Visalia & USA-CA & 107 & 36.30 & -119.22 & Jan 1983-Feb 2007 & BSk \\
\hline CA-14 & 035 & Bishop & USA-CA & 1271 & 37.36 & -118.41 & Feb 1983-Aug 2016 & BSk \\
\hline CA-15 & 039 & Parlier & USA-CA & 103 & 36.60 & -119.50 & May 1983-Aug 2016 & BSk \\
\hline CA-16 & 041 & Calipatria/Mulberry & USA-CA & -34 & 33.04 & -115.42 & Jul 1983-Aug 2016 & BWh \\
\hline CA-17 & 043 & McArthur & USA-CA & 1009 & 41.06 & -121.46 & Dec 1983-Aug 2016 & $\mathrm{Csb}$ \\
\hline CA-18 & 044 & U.C.Riverside & USA-CA & 311 & 33.96 & -117.34 & Jun 1985-Aug 2016 & BSk \\
\hline CA-19 & 047 & Brentwood & USA-CA & 14 & 37.93 & -121.66 & Nov 1985-Aug 2016 & $\mathrm{Csb}$ \\
\hline CA-20 & 049 & Oceanside & USA-CA & 15 & 33.26 & -117.32 & Mar 1986-Oct 2003 & BSk \\
\hline CA-21 & 054 & Blackwells Corner & USA-CA & 215 & 35.65 & -119.96 & Oct 1986-Aug 2016 & BWk \\
\hline CA-22 & 056 & Los Banos & USA-CA & 29 & 37.10 & -120.75 & Jun 1988-Aug 2016 & BSk \\
\hline CA-23 & 061 & Orland & USA-CA & 60 & 39.69 & -122.15 & May 1987-May 2010 & Csa \\
\hline CA-24 & 062 & Temecula & USA-CA & 433 & 33.49 & -117.23 & Nov 1986-Aug 2016 & BSk \\
\hline CA-25 & 064 & Santa Ynez & USA-CA & 149 & 34.58 & -120.08 & Nov 1986-Aug 2016 & $\mathrm{Csb}$ \\
\hline CA-26 & 068 & Seeley & USA-CA & 12 & 32.76 & -115.73 & May 1987-Aug 2016 & BWh \\
\hline CA-27 & 070 & Manteca & USA-CA & 10 & 37.83 & -121.22 & Nov 1987-Aug 2016 & BSk \\
\hline CA-28 & 071 & Modesto & USA-CA & 11 & 37.65 & -121.19 & Jul 1987-Aug 2016 & $\mathrm{BSk}$ \\
\hline CA-29 & 077 & Oakville & USA-CA & 58 & 38.43 & -122.41 & Jan 1989-Aug 2016 & $\mathrm{Csb}$ \\
\hline CA-30 & 075 & Irvine & USA-CA & 125 & 33.69 & -117.72 & Oct 1987-Aug 2016 & $\mathrm{BSk}$ \\
\hline CA-31 & 078 & Pomona & USA-CA & 223 & 34.06 & -117.81 & Mar 1989-Aug 2016 & $\mathrm{Csa}$ \\
\hline CA-32 & 080 & Fresno State & USA-CA & 103 & 36.82 & -119.74 & Oct 1988-Aug 2016 & BSk \\
\hline CA-33 & 083 & Santa Rosa & USA-CA & 24 & 38.40 & -122.80 & Jan 1990-Aug 2016 & $\mathrm{Csb}$ \\
\hline CA-34 & 084 & Browns Valley & USA-CA & 287 & 39.25 & -121.32 & Apr 1989-Aug 2016 & Csa \\
\hline CA-35 & 085 & Hopland F.S. & USA-CA & 354 & 39.01 & -123.08 & Sep 1989-Apr 2016 & Csa \\
\hline CA-36 & 086 & Lindcove & USA-CA & 146 & 36.36 & -119.06 & May 1989-Aug 2016 & Csa \\
\hline CA-37 & 087 & Meloland & USA-CA & -15 & 32.81 & -115.45 & Dec 1989-Aug 2016 & BWh \\
\hline CA-38 & 088 & Cuyama & USA-CA & 698 & 34.94 & -119.67 & May 1989-Aug 2016 & BSk \\
\hline CA-39 & 091 & Tulelake F.S. & USA-CA & 1230 & 41.96 & -121.47 & Mar 1989-Aug 2016 & Dsb \\
\hline CA-40 & 092 & Kesterson & USA-CA & 23 & 37.23 & -120.88 & Oct 1989-Aug 2016 & BSk \\
\hline CA-41 & 094 & Goletta foothills & USA-CA & 195 & 34.47 & -119.87 & Jul 1989-Jul 2016 & $\mathrm{Csb}$ \\
\hline CA-42 & 099 & Santa Monica & USA-CA & 104 & 34.04 & -118.48 & Dec 1992-Aug 2016 & $\mathrm{Csb}$ \\
\hline CA-43 & 103 & Windsor & USA-CA & 26 & 38.53 & -122.83 & Dec 1990-Aug 2016 & $\mathrm{Csb}$ \\
\hline CA-44 & 104 & De Laveaga & USA-CA & 91 & 37.00 & -122.00 & Sep 1990-Aug 2016 & $\mathrm{Csb}$ \\
\hline CA-45 & 105 & Westlands & USA-CA & 58 & 36.63 & -120.38 & Apr 1992-Aug 2016 & BWk \\
\hline CA-46 & 106 & Sanel Valley & USA-CA & 160 & 38.98 & -123.09 & Feb 1991-Aug 2016 & Csa \\
\hline CA-47 & 57 & Buntingville & USA-CA & 1221 & 40.29 & -120.43 & June 1986-Sep 2016 & Dsb \\
\hline CA-48 & 90 & Alturas & USA-CA & 1343 & 41.44 & -120.48 & Apr 1989-Sep 2016 & Dsb \\
\hline CA-49 & 151 & Ripley & USA-CA & 77 & 33.53 & -114.63 & Dec 1998-Sep 2016 & $\mathrm{BWh}$ \\
\hline CA-50 & 183 & Owens Lake North & USA-CA & 1123 & 36.49 & -117.92 & Dec 2002-Sep 2016 & BWk \\
\hline CA-51 & 147 & Otay Lake & USA-CA & 177 & 32.63 & -116.94 & Apr 1999-Sep 2016 & $\mathrm{Csb}$ \\
\hline CA-52 & 175 & Palo Verde II & USA-CA & 70 & 33.38 & -114.72 & Jan 2001-Sep 2016 & BWh \\
\hline CA-53 & 135 & Blynthe NE & USA-CA & 84 & 33.66 & -114.56 & Jan 1997-Sep 2016 & BWh \\
\hline CA-54 & 155 & Bryte & USA-CA & 12 & 38.60 & -121.54 & Dec 1998-Sep 2016 & Csa \\
\hline CA-55 & 159 & Monrovia & USA-CA & 181 & 34.15 & -117.99 & Oct $1999-$ Sep 2016 & Csa \\
\hline CA-56 & 161 & Patterson & USA-CA & 56 & 37.44 & -121.14 & Aug 1999-Sep 2016 & BSk \\
\hline
\end{tabular}


Table 1. Continued.

\begin{tabular}{|c|c|c|c|c|c|c|c|c|}
\hline No. & Code & Station & Country & $\begin{array}{r}\text { Elevation } \\
(\mathrm{m})\end{array}$ & $\begin{array}{r}\text { Lat } \\
\text { (Dec. deg.) }\end{array}$ & $\begin{array}{r}\text { Long } \\
\text { (Dec. deg.) }\end{array}$ & Period & $\begin{array}{l}\text { Köppen } \\
\text { class }^{\mathrm{a}}\end{array}$ \\
\hline CA-57 & 174 & Long Beach & USA-CA & 5 & 33.80 & -118.09 & Sep 2000-Sep 2016 & $\mathrm{Csb}$ \\
\hline CA-58 & 173 & Torrey Pines & USA-CA & 102 & 32.90 & -117.25 & Nov 2000-Sep 2016 & Csa \\
\hline CA-59 & 150 & Miramar & USA-CA & 136 & 32.89 & -117.14 & Apr 1999-Sep 2016 & Csa \\
\hline CA- 60 & 153 & Escondido SPV & USA-CA & 119 & 33.08 & -116.98 & Feb 1999-Sep 2016 & $\mathrm{Csb}$ \\
\hline A-1 & 32040 & Townsville Aero & Australia & 4 & -19.25 & 146.77 & $(1940 / 1996-2016)^{\mathrm{b}}$ & Aw \\
\hline A-2 & 33307 & Woolshed & Australia & 556 & -19.42 & 146.54 & $(1990 / 2003-2016)$ & Aw \\
\hline A-3 & 2056 & Kununurra Aero & Australia & 44 & -15.78 & 128.71 & (1971/1990-2016) & $\mathrm{BSh}$ \\
\hline A-4 & 35264 & Emerald & Australia & 189 & -23.57 & 148.18 & (1990/1998-2016) & $\mathrm{BSh}$ \\
\hline$A-5$ & 24024 & Loxton R.C. & Australia & 30 & -34.44 & 140.6 & (1984/1998-2016) & BSk \\
\hline A-6 & 74037 & Yanco AG.I. & Australia & 164 & -34.62 & 146.43 & (1957/1999-2016) & $\mathrm{BSk}$ \\
\hline A-7 & 74258 & Deniliquin Airp.AWS & Australia & 94 & -35.56 & 144.95 & (1990/2003-2016) & BSk \\
\hline A-8 & 75041 & Griffith Airp.AWS & Australia & 134 & -34.25 & 146.07 & (1958/1990-2016) & BSk \\
\hline A-9 & 76031 & Mildura Airp. & Australia & 50 & -34.24 & 142.09 & (1946/1993-2016) & BSk \\
\hline A-10 & 24048 & Renmark Apt.1 & Australia & 32 & -34.2 & 140.68 & (1990/2003-2016) & BWk \\
\hline A-11 & 40082 & University of QLD G. & Australia & 89 & -27.54 & 152.34 & (1897/1990-2016) & Cfa \\
\hline A-12 & 40922 & Kingaroy Airp. & Australia & 434 & -26.57 & 151.84 & (1990/2003-2016) & $\mathrm{Cfa}$ \\
\hline A-13 & 41359 & Oakey Aero & Australia & 406 & -27.4 & 151.74 & (1970/1996-2016) & Cfa \\
\hline A-14 & 41522 & Dalby Airp. & Australia & 344 & -27.16 & 151.26 & (1990/2006-2016) & $\mathrm{Cfa}$ \\
\hline A-15 & 41525 & Warwick & Australia & 475 & -28.21 & 152.1 & (1990/2000-2016) & $\mathrm{Cfa}$ \\
\hline A-16 & 41529 & Toowoomba Airp. & Australia & 641 & -27.54 & 151.91 & (1990/1997-2016) & $\mathrm{Cfa}$ \\
\hline A-17 & 80091 & Kyabram & Australia & 105 & -36.34 & 145.06 & (1964/1990-2016) & $\mathrm{Cfa}$ \\
\hline A-18 & 81049 & Tatura I.S.A. & Australia & 114 & -36.44 & 145.27 & (1942/1990-2016) & $\mathrm{Cfa}$ \\
\hline A-19 & 81124 & Yarrawonga & Australia & 129 & -36.03 & 146.03 & $(1990 / 2003-2016)$ & $\mathrm{Cfa}$ \\
\hline A-20 & 81125 & Shepparton Airp. & Australia & 114 & -36.43 & 145.39 & (1990/1996-2016) & $\mathrm{Cfa}$ \\
\hline A-21 & 41175 & Applethorpe & Australia & 872 & -28.62 & 151.95 & (1966/2006-2016) & $\mathrm{Cfb}$ \\
\hline A-22 & 81123 & Bendigo Airp. & Australia & 208 & -36.74 & 144.33 & (1990/2004-2016) & $\mathrm{Cfb}$ \\
\hline A-23 & 85072 & East Sale Airp. & Australia & 5 & -38.12 & 147.13 & (1943/1996-2016) & $\mathrm{Cfb}$ \\
\hline A-24 & 85279 & Bairnsdale Airp. & Australia & 49 & -37.88 & 147.57 & (1942/2003-2016) & $\mathrm{Cfb}$ \\
\hline A-25 & 85280 & Morwell L.V.Airp. & Australia & 56 & -38.21 & 146.47 & (1984/1999-2016) & $\mathrm{Cfb}$ \\
\hline A-26 & 85296 & Mount Moornapa & Australia & 480 & -37.75 & 147.14 & (1990/2003-2016) & $\mathrm{Cfb}$ \\
\hline A-27 & 90035 & Colac & Australia & 261 & -38.23 & 143.79 & (1990/2003-2016) & $\mathrm{Cfb}$ \\
\hline A- 28 & 9538 & Dwellingup & Australia & 267 & -32.71 & 116.06 & (1934/1990-2016) & $\mathrm{Csb}$ \\
\hline A-29 & 9617 & Bridgetown & Australia & 179 & -33.95 & 116.13 & (1990/2003-2016) & $\mathrm{Csb}$ \\
\hline A-30 & 23373 & Nuriootpa Pirsa & Australia & 275 & -34.48 & 139.01 & (1990/1996-2016) & $\mathrm{Csb}$ \\
\hline A-31 & 26021 & Mount Gambier Aero & Australia & 63 & -37.75 & 140.77 & (1942/1994-2016) & $\mathrm{Csb}$ \\
\hline A-32 & 26091 & Coonawarra & Australia & 57 & -37.29 & 140.83 & (1985/1990-2016) & $\mathrm{Csb}$ \\
\hline A-33 & 66062 & Sydney (Obs.Hill) & Australia & 39 & -33.86 & 151.21 & (1858/1990-2016) & $\mathrm{Cfb}$ \\
\hline A-34 & 33002 & Ayr DPI Res.St. & Australia & 17 & -19.62 & 147.38 & (1951/1994-2016) & Cwa \\
\hline A-35 & 7176 & Newman Aero & Australia & 524 & -23.42 & 119.8 & (1971/2003-2016) & BWh \\
\hline A-36 & 13017 & Giles & Australia & 598 & -25.03 & 128.3 & (1956/1990-2016) & BWh \\
\hline A-37 & 11052 & Forrest & Australia & 159 & -30.85 & 128.11 & (1990/2003-2016) & $\mathrm{BWh}$ \\
\hline A-38 & 11003 & Eucla & Australia & 93 & -31.68 & 128.9 & (1876/1995-2016) & BSk \\
\hline A-39 & 12071 & Salmon Gums & Australia & 249 & -32.99 & 121.62 & (1932/2003-2016) & BSk \\
\hline A-40 & 7045 & Meekatharra Airp. & Australia & 517 & -26.61 & 118.54 & (1944/1992-2016) & $\mathrm{BWh}$ \\
\hline A-41 & 1025 & Doongan & Australia & 385 & -15.38 & 126.31 & (1988/1990-2016) & Aw \\
\hline A-42 & 2012 & Halls Creek Airp. & Australia & 422 & -18.23 & 127.66 & (1944/1996-2016) & BSh \\
\hline A-43 & 13015 & Carnegie & Australia & 448 & -25.8 & 122.98 & (1942/1990-2016) & $\mathrm{BWh}$ \\
\hline A-44 & 3080 & Curtin Aero & Australia & 78 & -17.58 & 123.83 & (1990/2003-2016) & $\mathrm{BSh}$ \\
\hline A-45 & 6022 & Gascoyne Junction & Australia & 144 & -25.05 & 115.21 & (1907/1990-2016) & BWh \\
\hline A-46 & 9789 & Esperance & Australia & 25 & -33.83 & 121.89 & (1969/1990-2016) & Csb \\
\hline A-47 & 91223 & Marrawah & Australia & 107 & -40.91 & 144.71 & (1971/1990-2016) & $\mathrm{Cfb}$ \\
\hline A-48 & 18106 & Nullarbor & Australia & 64 & -31.45 & 130.9 & (1888/2006-2016) & BWk \\
\hline A-49 & 16090 & Coober Pedy Airp. & Australia & 225 & -29.03 & 134.72 & (1990/2004-2016) & $\mathrm{BWh}$ \\
\hline A-50 & 16085 & Marla Police St. & Australia & 323 & -27.3 & 133.62 & (1985/1990-2016) & $\mathrm{BWh}$ \\
\hline A-51 & 13011 & Warburton Airfield & Australia & 459 & -26.13 & 126.58 & (1940/2003-2016) & BWh \\
\hline
\end{tabular}


Table 1. Continued.

\begin{tabular}{|c|c|c|c|c|c|c|c|c|}
\hline No. & Code & Station & Country & $\begin{array}{r}\text { Elevation } \\
(\mathrm{m})\end{array}$ & $\begin{array}{r}\text { Lat } \\
\text { (Dec. deg.) }\end{array}$ & $\begin{array}{r}\text { Long } \\
\text { (Dec. deg.) }\end{array}$ & Period & $\begin{array}{l}\text { Köppen } \\
\text { class }^{a}\end{array}$ \\
\hline$A-52$ & 15528 & Yuendumu & Australia & 667 & -22.26 & 131.8 & (1952/1990-2016) & BWh \\
\hline A-53 & 15666 & Rabbit Flat & Australia & 340 & -20.18 & 130.01 & (1990/1996-2016) & BWh \\
\hline A-54 & 14829 & Lajamanu Airp. & Australia & 316 & -18.33 & 130.64 & (1952/1990-2016) & $\mathrm{BSh}$ \\
\hline$A-55$ & 15135 & Tennant Creek Airp. & Australia & 376 & -19.64 & 134.18 & (1969/1992-2016) & $\mathrm{BSh}$ \\
\hline A-56 & 37010 & Camooweal Township & Australia & 231 & -19.92 & 138.12 & (1891/2003-2016) & BWh \\
\hline$A-57$ & 14707 & Wollogorang & Australia & 60 & -17.21 & 137.95 & (1967/1990-2016) & Aw \\
\hline A-58 & 14938 & Mango Farm & Australia & 15 & -13.74 & 130.68 & (1980/1990-2016) & Aw \\
\hline A-59 & 69134 & Batemans Bay & Australia & 11 & -35.72 & 150.19 & (1985/1991-2016) & $\mathrm{Cfb}$ \\
\hline A-60 & 14198 & Jabiru Airp. & Australia & 27 & -12.66 & 132.89 & (1971/1990-2016) & Aw \\
\hline A-61 & 28008 & Lockhart River Airp. & Australia & 19 & -12.79 & 143.3 & (1956/2001-2016) & Am \\
\hline A-62 & 34084 & Charters Towers Airp. & Australia & 290 & -20.05 & 146.27 & (1990/1992-2016) & BSh \\
\hline A-63 & 29038 & Kowanyama Airp. & Australia & 10 & -15.48 & 141.75 & (1912/1999-2016) & Aw \\
\hline A-64 & 32078 & Ingham Composite & Australia & 12 & -18.65 & 146.18 & $(1968 / 1990-2016)$ & $\mathrm{Am}$ \\
\hline A-65 & 40854 & Logan City W.T.P. & Australia & 14 & -27.68 & 153.19 & (1990/1992-2016) & Cfa \\
\hline A-66 & 8095 & Mullewa & Australia & 268 & -28.54 & 115.51 & (1896/1990-2016) & BSh \\
\hline A-67 & 8251 & Kalbarri & Australia & 6 & -27.71 & 114.17 & (1970/1990-2016) & BSh \\
\hline A-68 & 8225 & Eneabba & Australia & 100 & -29.82 & 115.27 & (1964/1990-2016) & Csa \\
\hline A-69 & 7139 & Paynes Find & Australia & 339 & -29.27 & 117.68 & (1919/1990-2016) & BWh \\
\hline A-70 & 10007 & Bencubbin & Australia & 359 & -30.81 & 117.86 & $(1912 / 1990-2016)$ & $\mathrm{BSh}$ \\
\hline A-71 & 10092 & Merredin & Australia & 315 & -31.48 & 118.28 & $(1903 / 1990-2016)$ & BSk \\
\hline A-72 & 12038 & Kalgoorlie-Boulder Airp. & Australia & 365 & -30.78 & 121.45 & (1939/1994-2016) & $\mathrm{BSh}$ \\
\hline A-73 & 16098 & Tarcoola Aero & Australia & 123 & -30.71 & 134.58 & (1990/1999-2016) & BWh \\
\hline A-74 & 18195 & Minnipa Pirsa & Australia & 165 & -32.84 & 135.15 & (1990/2003-2016) & BSk \\
\hline A-75 & 46126 & Tibooburra Airp. & Australia & 176 & -29.44 & 142.06 & (1990/2003-2016) & BWh \\
\hline A-76 & 48245 & Boorke Airp. AWS & Australia & 107 & -30.04 & 145.95 & $(1990 / 2002-2016)$ & $\mathrm{BSh}$ \\
\hline A-77 & 55325 & Tamworth Airp. AWS & Australia & 395 & -31.07 & 150.84 & (1990/2006-2016) & Cfa \\
\hline A-78 & 38026 & Birdsville Airp. & Australia & 47 & -25.9 & 139.35 & (1990/2001-2016) & BWh \\
\hline A-79 & 30161 & Richmond Airp. & Australia & 206 & -20.7 & 143.12 & (1990/2003-2016) & $\mathrm{BSh}$ \\
\hline A- 80 & 33013 & Collinsville Airp. & Australia & 196 & -20.55 & 147.85 & (1939/1990-2016) & BSh \\
\hline
\end{tabular}

${ }^{a}$ Köppen classification obtained from Peel et al. (2007). ${ }^{b}$ In the case of Australian stations, the periods of observations vary between different climatic parameters; e.g. for the case (1939/1990-2016), the two dates separated by a slash show the starting date of the oldest and newest record of parameters used in calculations, while 2016 is the end date of the records.

In the case of CIMIS stations, the monthly data for all climatic parameters were obtained, including $\mathrm{ET}_{\mathrm{o}}$ estimations using the CIMIS method (Snyder and Pruitt, 1985, 1992), but they required quality control before their use. Quality control signs are provided by the database for all climatic data, indicating extreme values, while possible errors are flagged but they are not automatically excluded. For this reason, the user should consider the signs in order to prepare a robust dataset. For this study, proper control was performed and very extreme or erroneous monthly values were excluded. Excluded values were less than $1 \%$ of the total values of all stations and all parameters. The final clean dataset was subjected to a secondary but indirect quality control through the comparison between the estimated mean monthly values of $\mathrm{ET}_{\mathrm{o}}$ of the ASCE-short method (Eq. 1) using the clean climatic data of all USA-CA stations versus the respective mean monthly $\mathrm{ET}_{\mathrm{o}}$ values given by the CIMIS database (linear regression result between mean monthly values for $n$ obs. $=12 \times 60=720: y=0.994 x-1.07$ with $R^{2}=0.98$ ) (see Fig. S1 in the Supplement). Data cleaning was not fol- lowed in the case of Australian stations, since the AGBM database provides the mean monthly values of the climatic parameters for the total periods of observation and not for individual years. The general statistics of the mean monthly observed values of climatic parameters obtained from the 140 stations of California (USA) and Australia are given in Table $\mathrm{S} 1$ of the Supplement. A comparison of the parameters $T_{\max }, T_{\min }, R_{\mathrm{S}}, R_{\mathrm{n}}, \mathrm{DE}$ and $u_{2}$ between the rasters $\left(0.5^{\circ}\right)$ and the station data are provided in Fig. S2a, b, c, d, e and f of the Supplement.

The validation procedure was performed using the data of the stations in Table 1 by comparing the mean monthly values of $\mathrm{ET}_{\mathrm{o}}$ derived by the P-T (Eq. 2) and H-S (Eq. 4b, c) methods with the standard $a_{\mathrm{pt}}$ and $c_{\mathrm{hs} 2}$ coefficients and with the readjusted ones versus the ASCE method for the short reference crop (Eq. 1). The same procedure was also performed for the new $a_{\mathrm{pt}}$ and $c_{\mathrm{hs} 2}$ coefficients for the tall reference crop and for the readjusted coefficient $K_{\mathrm{RS}}$ in the radiation formula of H-S (Eq. 3). For the case of the ASCE method for the short reference crop, additional models of re- 

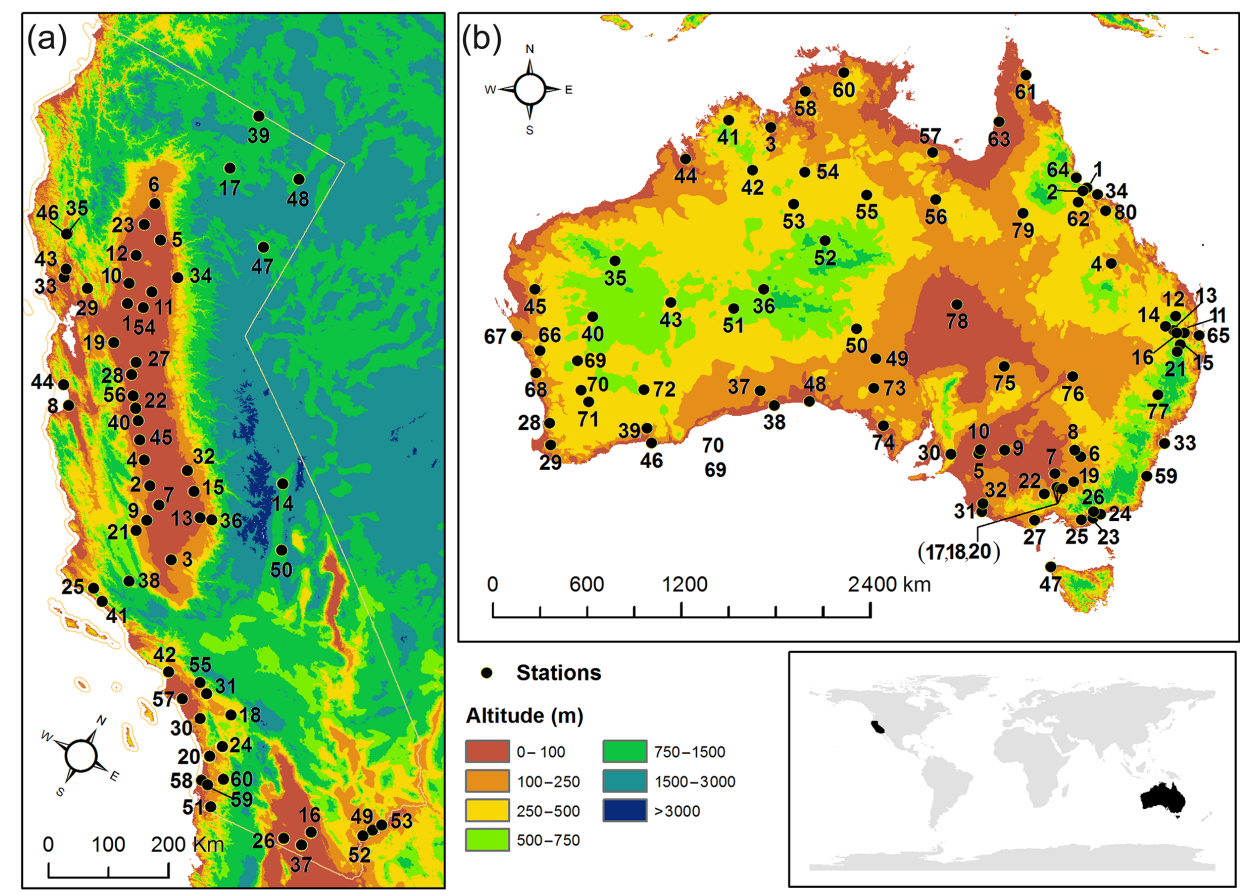

Figure 1. Position of stations (a) from California (USA) obtained from the CIMIS database and (b) from Australia obtained from AGBM database (the station numbers are those from Table 1 without the abbreviations CA- and A-).

duced parameters were used from the literature in order to perform comparisons with the standard and readjusted P-T and $\mathrm{H}-\mathrm{S}$ models. The selection of these models was made in such way as to satisfy the following criteria/characteristics:

- The selected models have been calibrated by either using global data or a representative amount of data from California or Australia. Models that have been tested for California and Australia and showed good performance were also included.

- The selected models showed better performance in comparison to other models when tested using the validation datasets of Californian and Australian stations, but they also showed good performance with regard to other regions based on studies from the literature. It has to be mentioned that an extremely large amount of models were examined taking into account the modified H-S and P-T models obtained from literature that has already been cited in the introduction and the large lists of models presented in the work of Valipour (2015a, b, 2017) and Valipour et al. (2017). Strict modifications of the $\mathrm{P}-\mathrm{T}$ and $\mathrm{H}-\mathrm{S}$ models with fixed coefficients calibrated for local conditions were not used because they cannot adapt their coefficients to the large climatic variability in the validation dataset.

- The majority of the selected models require additional parameters in comparison to the P-T and H-S models. This criterion was used in order to compare the strength of the readjusted P-T and H-S coefficients versus such models.

Based on the aforementioned criteria, the following eight models were selected for comparisons with the standard and readjusted $\mathrm{H}-\mathrm{S}$ and P-T models (Table 2):

- Two modified models of $\mathrm{H}-\mathrm{S}$ by Droogers and Allen (2002), where the second one uses precipitation as an additional parameter. The models were calibrated using global data.

- Three models of reduced parameters given by Valiantzas (2013a, b, 2014), which were calibrated using 535 stations from Europe, Asia and Africa. The first model uses temperature and radiation data, while the other two use temperature, radiation and humidity data. The models have been tested for Californian (Valiantzas, 2013c) and Australian conditions (Ahooghalandari et al., 2017).

- Two models of reduced parameters by Ahooghalandari et al. (2016) calibrated/validated using stations from various locations in Australia. The models use temperature and relative humidity data.

- The Copais model of Alexandris et al. (2006) that uses temperature, radiation and humidity data. The model was calibrated/validated using data from Greece, California and Oregon (USA), while it has shown a very 


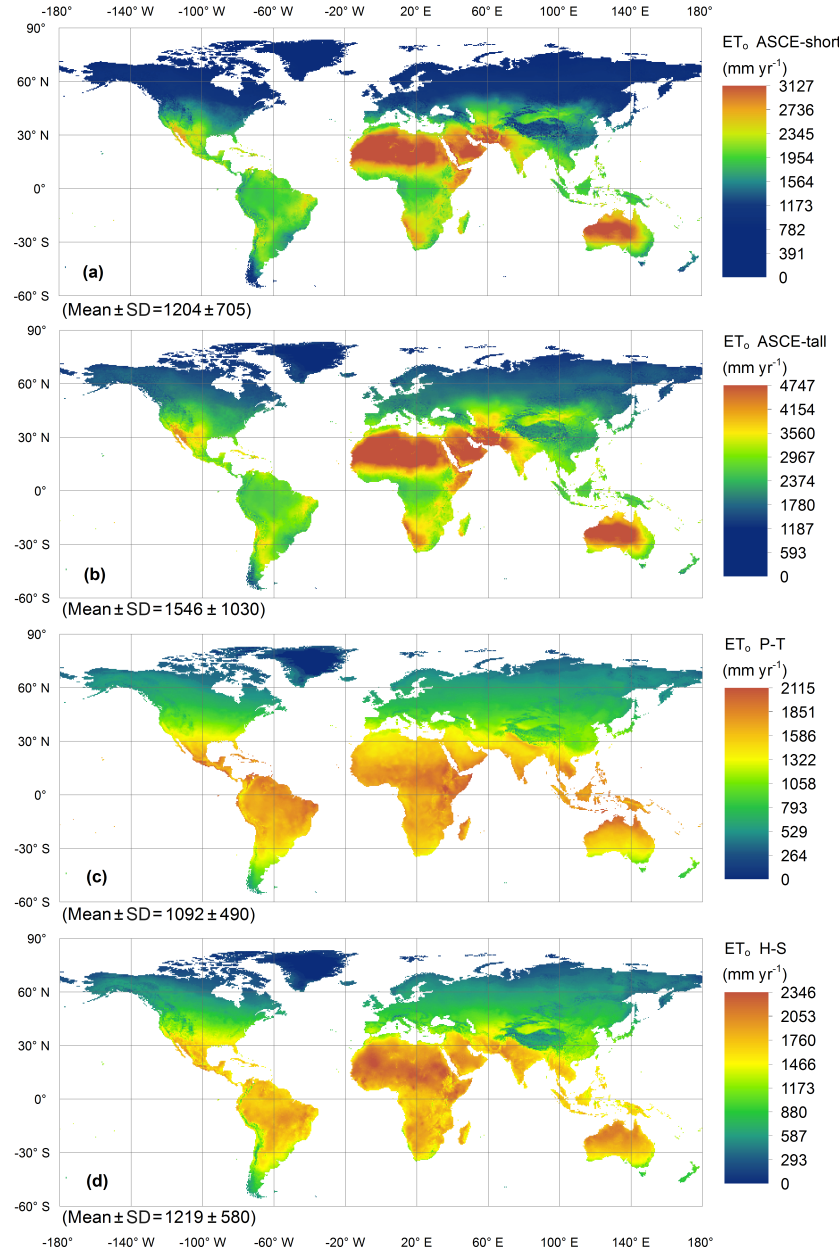

Figure 2. Mean annual values $\left(\mathrm{mm} \mathrm{year}^{-1}\right)$ of $\mathrm{ET}_{\mathrm{o}}$ for the period 1950-2000 using (a) the ASCE-short method, (b) the ASCE-tall method, (c) the standard P-T method for $a_{\mathrm{pt}}=1.26$ and (d) the standard H-S method for $c_{\mathrm{hs} 2}=0.0023\left(0.5^{\circ}\right.$ resolution maps; mean \pm SD are estimated after conversion from WGS84 to Cylindrical Equal Area coordinate system).

good response to many other regions of the world including Australia (Ahooghalandari et al., 2017).

The following statistical criteria were used in the validation procedure: the coefficient of determination $\left(R^{2}\right)$, the modified coefficient of determination $\left(b R^{2}\right)$ based on $y=$ $b x$ (Krause et al., 2005), mean absolute error (MAE), root mean square error (RMSE), percent bias (PBIAS \%), the Nash-Sutcliffe efficiency (NSE; Nash and Sutcliffe, 1970), the index of agreement ( $d$; Willmott, 1981) and the KlingGupta efficiency (KGE; Gupta et al., 2009). The criteria were calculated using the package $\{$ HydroGOF $\}$ in $\mathrm{R}$ language (Zambrano-Bigiarini, 2015; see the package manual for formulas).

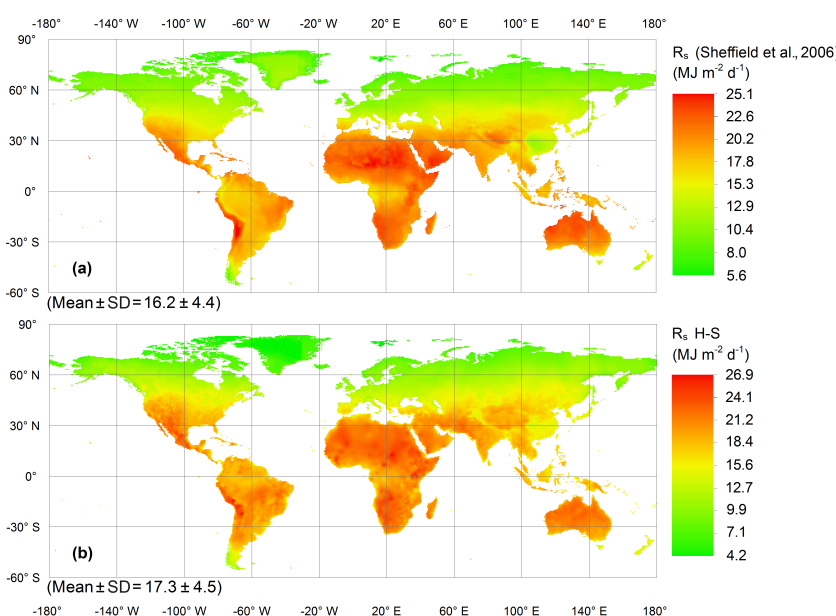

Figure 3. Mean annual values of $R_{\mathrm{S}}\left(\mathrm{MJ} \mathrm{m}^{-2} \mathrm{~d}^{-1}\right)$ for the period 1950-2000 (a) from the database of Sheffield et al. (2006) and (b) estimated using the standard $\mathrm{H}-\mathrm{S}$ radiation formula for $K_{\mathrm{RS}}=0.17$ (Eq. 3) $\left(0.5^{\circ}\right.$ resolution maps; mean $\pm \mathrm{SD}$ are estimated after conversion from WGS84 to Cylindrical Equal Area coordinate system).

\section{Results}

\subsection{Comparative analysis between the standard $\mathrm{ET}_{\mathrm{O}}$ formulas of ASCE, P-T and H-S and error analysis of the $\mathrm{H}-\mathrm{S}$ radiation formula}

The global maps of mean monthly $\mathrm{ET}_{\mathrm{o}}$ at $0.5^{\circ}$ resolution for the period 1950-2000 using (a) the methods of ASCE (Eq. 1) for both reference crops (ASCE-short and ASCE-tall), (b) the standard P-T method (Eq. 2) for $a_{\mathrm{pt}}=1.26$ and (c) the standard H-S method (Eq. $4 \mathrm{~b}$ ) for $c_{\mathrm{hs} 2}=0.0023$ were developed. The respective mean annual $\mathrm{ET}_{\mathrm{o}}$ maps are given in Fig. 2a, $\mathrm{b}$, c and d. Similarly, the mean annual $R_{\mathrm{S}}$ values provided by Sheffield et al. (2006) and the corresponding $R_{\mathrm{S}}$ values estimated by the standard H-S radiation formula (Eq. 3 with $\left.K_{\mathrm{RS}}=0.17\right)$ are given in Fig. 3a and b, respectively.

The MAD \% (Eq. 5) maps of the ASCE-tall, standard P$\mathrm{T}$ and standard H-S methods versus ASCE-short are given in Fig. 4a, b and c, while the MAD\% of the standard solar radiation formula of $\mathrm{H}-\mathrm{S}$ versus the $R_{\mathrm{S}}$ values given by Sheffield et al. (2006) is also given in Fig. 4d. The percentage globe coverage (excluding Antarctica) for different classes of MAD \% and the $R^{2}$ and RMSD based on respective comparisons of the mean monthly values of $\mathrm{ET}_{\mathrm{o}}$ and $R_{\mathrm{S}}$ methods are given in Table 3.

The case of MAD \% between the $\mathrm{ET}_{0}$ methods of ASCEtall and ASCE-short (Fig. 4a and Table 3) indicates that there is $25.2 \%$ map coverage in the MAD \% class of $\pm 10 \%$ where the effects of reference crop type are significantly minimized (Table 3). These territories include the regions of tropical rainforests in Latin America, central Africa and Indonesia, regions of large mountain formations and ranges of high ele- 
Table 2. Additional models of reduced parameters obtained from the international literature, which provide equivalent results to $\mathrm{ET}_{\mathrm{O}}$ for the short reference crop.

\begin{tabular}{|c|c|c|c|}
\hline Reference & Abbreviation & Formula & Climate data requirements* \\
\hline Droogers and Allen (2002) & DRAL1 (Eq. 8) & $\mathrm{ET}_{\mathrm{o}}=0.00102 R_{\mathrm{a}}\left(T_{\text {mean }}+16.8\right) \cdot(\mathrm{TD})^{0.5}$ & $T_{\max }, T_{\min }$ \\
\hline Droogers and Allen (2002) & DRAL2 (Eq. 9) & $\begin{aligned} \mathrm{ET}_{\mathrm{o}}= & 0.0005304 R_{\mathrm{a}}\left(T_{\text {mean }}+17.0\right) \\
& (\mathrm{TD}-0.0123 P)^{0.76}\end{aligned}$ & $T_{\max }, T_{\min }, P$ \\
\hline Alexandris et al. (2006) & Copais (Eq. 10) & $\begin{array}{l}\mathrm{ET}_{\mathrm{o}}=0.057+0.227 C_{2}+0.643 C_{1}+0.0124 C_{1} C_{2} \\
C_{1}=0.6416-0.00784 \mathrm{RH}+0.372 R_{\mathrm{S}}-0.00264 R_{\mathrm{S}} \mathrm{RH} \\
C_{2}=-0.0033+0.00812 T_{\text {mean }}+0.101 R_{\mathrm{S}}+0.00584 R_{\mathrm{S}} T_{\text {mean }}\end{array}$ & $T_{\text {mean }}, R_{\mathrm{s}}, \mathrm{RH}$ \\
\hline Valiantzas (2013a, 2014) & VAL1 (Eq. 11) & $\begin{aligned} \mathrm{ET}_{\mathrm{o}} & =0.0393 R_{\mathrm{S}} \sqrt{T_{\text {mean }}+9.5}-0.19 R_{\mathrm{s}}^{0.6} \varphi^{0.15} \\
& +0.0061\left(T_{\text {mean }}+20\right)\left(1.12 T_{\text {mean }}-T_{\text {min }}-2\right)^{0.7}\end{aligned}$ & $T_{\text {mean }}, T_{\min }, R_{\mathrm{s}}$ \\
\hline Valiantzas (2013a, 2014) & VAL2 (Eq. 12) & $\begin{aligned} \mathrm{ET}_{\mathrm{O}} & =0.0393 R_{\mathrm{S}} \sqrt{T_{\text {mean }}+9.5}-0.19 R_{\mathrm{S}}^{0.6} \varphi^{0.15} \\
& +0.078\left(T_{\text {mean }}+20\right)\left(1-\frac{\mathrm{RH}}{100}\right)\end{aligned}$ & $T_{\text {mean }}, R_{\mathrm{S}}, \mathrm{RH}$ \\
\hline Valiantzas (2013b) & VAL3 (Eq. 13) & $\begin{aligned} \mathrm{ET}_{\mathrm{o}} & =0.0393 R_{\mathrm{S}} \sqrt{T_{\text {mean }}+9.5}-2.4\left(\frac{R_{\mathrm{S}}}{R_{\mathrm{a}}}\right)^{2} \\
& +\mathrm{Cu}\left(T_{\text {mean }}+20\right)\left(1-\frac{\mathrm{RH}}{100}\right)\end{aligned}$ & $\begin{array}{l}T_{\text {mean }}, R_{\mathrm{s}}, \mathrm{RH} \\
(\mathrm{Cu}=0.054 \text { for } \mathrm{RH}>65 \% \text { and } \\
\mathrm{Cu}=0.083 \text { for } \mathrm{RH} \leq 65 \%)\end{array}$ \\
\hline Ahooghalandari et al. (2016) & AKJ1 (Eq. 14) & $\mathrm{ET}_{\mathrm{o}}=0.252 \cdot 0.408 R_{\mathrm{a}}+0.221 T_{\text {mean }}\left(1-\frac{\mathrm{RH}}{100}\right)$ & $T_{\text {mean }}, \mathrm{RH}$ \\
\hline Ahooghalandari et al. (2016) & AKJ2 (Eq. 15) & $\mathrm{ET}_{\mathrm{o}}=0.29 \cdot 0.408 R_{\mathrm{a}}+0.15 T_{\max }\left(1-\frac{\mathrm{RH}}{100}\right)$ & $T_{\max }, \mathrm{RH}$ \\
\hline
\end{tabular}

Table 3. The percent coverage ${ }^{\mathrm{a}}$ of MAD \% classes based on mean annual values (according to Fig. 4), $R^{2}$ and RMSD based on comparisons of the mean monthly values of $\mathrm{ET}_{\mathrm{O}}$ and $R_{\mathrm{S}}$ methods (comparisons based on $0.5^{\circ}$ resolution maps).

\begin{tabular}{lrrrr}
\hline $\begin{array}{l}\text { MAD } \% \\
\text { range }\end{array}$ & $\begin{array}{r}\mathrm{ET}_{\mathrm{o}}{ }^{\mathrm{b}}(\mathrm{ASCE}-\text { tall) for } \\
C_{\mathrm{n}}=1600, C_{\mathrm{d}}=0.38(\text { Eq. 1) }\end{array}$ & $\begin{array}{r}\mathrm{ET}_{\mathrm{o}}^{\mathrm{b}}(\mathrm{P}-\mathrm{T}) \text { for } \\
a_{\mathrm{pt}}=1.26(\mathrm{Eq} .2)\end{array}$ & $\begin{array}{r}\mathrm{ET}_{\mathrm{o}}^{\mathrm{b}}(\mathrm{H}-\mathrm{S}) \text { for } \\
c_{\mathrm{hs} 2}=0.0023 \text { (Eq. 4b) }\end{array}$ & $\begin{array}{r}R_{\mathrm{S}}{ }^{\mathrm{c}}(\mathrm{H}-\mathrm{S}) \text { for } \\
K_{\mathrm{RS}}=0.17(\text { Eq. 3) }\end{array}$ \\
\hline$\leq-50 \%$ & $0.0 \%{ }^{\mathrm{a}}$ & $0.8 \%$ & $0.0 \%$ & $1.0 \%$ \\
-50 up to $-25 \%$ & $0.0 \%$ & $14.8 \%$ & $5.2 \%$ & $2.2 \%$ \\
-25 up to $-10 \%$ & $0.0 \%$ & $10.8 \%$ & $15.4 \%$ & $7.1 \%$ \\
-10 up to $10 \%$ & $25.2 \%$ & $21.3 \%$ & $24.8 \%$ & $55.3 \%$ \\
10 up to 25\% & $40.9 \%$ & $22.5 \%$ & $19.6 \%$ & $32.8 \%$ \\
25 up to 50\% & $33.6 \%$ & $21.9 \%$ & $29.2 \%$ & $1.6 \%$ \\
$>50 \%$ & $0.3 \%$ & $7.9 \%$ & $5.8 \%$ & $0.0 \%$ \\
\hline$R^{2}$ & 0.98 & 0.77 & 0.89 & 0.92 \\
RMSD & $39.6^{\mathrm{d}}$ & $36.0^{\mathrm{d}}$ & $24.5^{\mathrm{d}}$ & $2.4^{\mathrm{e}}$ \\
\hline
\end{tabular}

a The percent coverage was estimated after conversion from WGS84 ellipsoid to projected Cylindrical Equal Area coordinate system without considering Antarctica. ${ }^{\mathrm{b}} \mathrm{MAD} \%$ of the three $\mathrm{ET}_{\mathrm{o}}$ methods is estimated versus ASCE-short. ${ }^{\mathrm{c}} \mathrm{MAD} \%$ of the standard solar radiation method of $\mathrm{H}-\mathrm{S}$ is estimated versus the $R_{\mathrm{S}}$ data of Sheffield et al. (2006). ${ }^{\mathrm{d}}$ The unit of RMSD for $\mathrm{ET}_{\mathrm{o}}$ is $\mathrm{mm}$ month ${ }^{-1}$. e The unit of RMSD for $R_{\mathrm{S}}$ is MJ m ${ }^{-2} \mathrm{~d}^{-1}$.

vation, and the regions of taigas and tundras of North America and Asia (Fig. 4a). The low values of the vapour pressure deficit are the main characteristic of these regions. By contrast, the largest differences between the two reference crops appear in arid and semi-arid environments due to the high values of the vapour pressure deficit. The high correlation $R^{2}=0.98$ (Table 3) between the mean monthly $\mathrm{ET}_{\mathrm{o}}$ values of ASCE-tall and ASCE-short suggests that it is feasible to develop reliable regional monthly coefficients or regression models, which can convert the $\mathrm{ET}_{\mathrm{o}}$ estimations from the short to the tall reference crop especially when the $\mathrm{ET}_{0}$ of the short reference crop is estimated with a method of reduced parameters (e.g. P-T or H-S) (a paradigm has been presented by Aschonitis et al., 2012).

Even though MAD $\%, R^{2}$ and RMSD for the standard P-T and H-S methods (Fig. 4b and c; Table 3) indicate a better performance of the second one to approximate the ASCEshort on a global scale, both methods seem to be equally valuable because their proximity to ASCE-short is maximized at relatively different climatic regions. This is indi- 


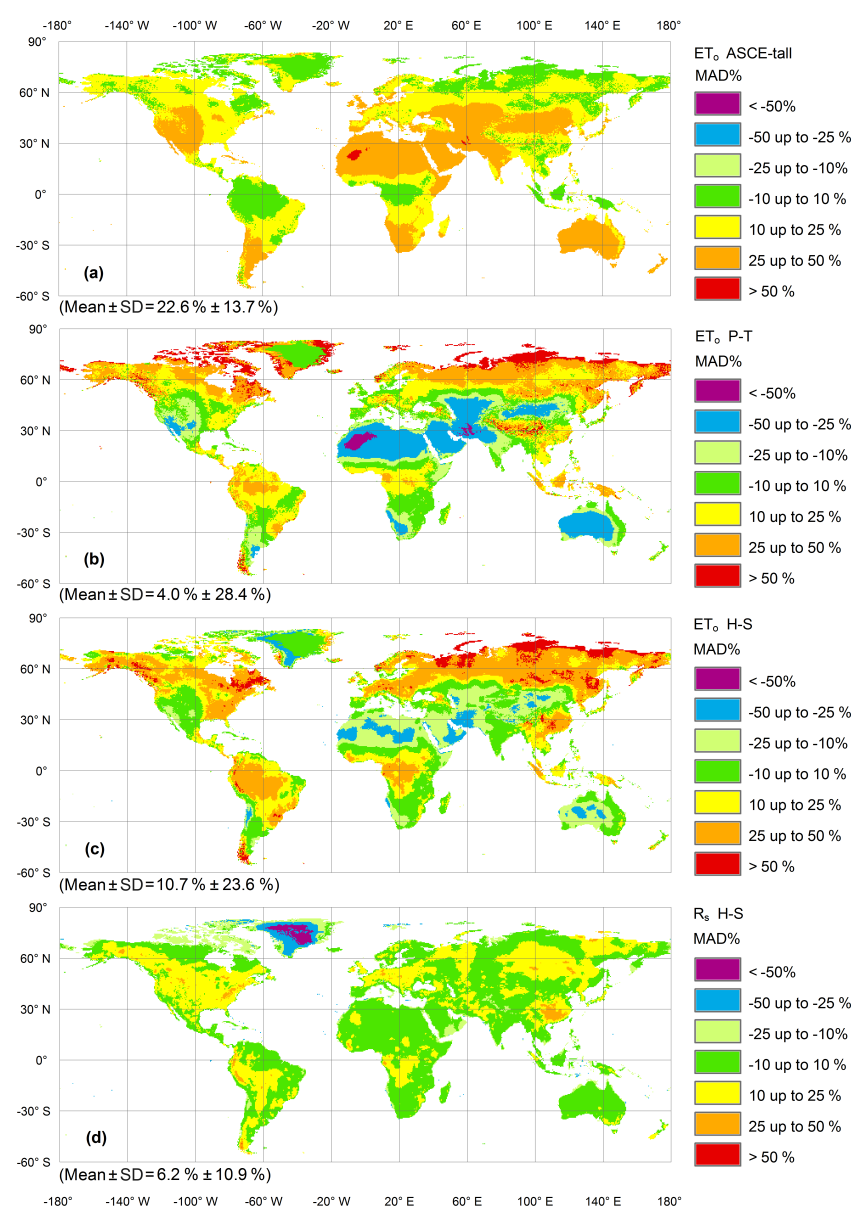

Figure 4. Mean annual difference percentage (MAD\%) of $\mathrm{ET}_{\mathrm{O}}$ between the ASCE-short and (a) the ASCE-tall method, (b) the standard P-T method for $a_{\mathrm{pt}}=1.26$, (c) the standard H-S method for $c_{\mathrm{hs} 2}=0.0023$, and (d) MAD \% between $R_{\mathrm{S}}$ values of Sheffield et al. (2006) and the standard solar radiation formula of H-S for $K_{\mathrm{RS}}=0.17\left(0.5^{\circ}\right.$ resolution maps; mean $\pm \mathrm{SD}$ are estimated after conversion from WGS84 to Cylindrical Equal Area coordinate system).

cated by the difference between the absolute MAD \% values (DMAD) (Eq. 6) of the P-T and H-S methods (Fig. 5a). The interpretation of Fig. 5a was performed using as a base the major climatic groups (CGs) of the Köppen-Geiger climate map in Peel et al. (2007) (Fig. 5b). The spatial extent of the major CGs of the Köppen-Geiger climate classification (without Antarctica) and the respective extent of DMAD classes (P-T versus $\mathrm{H}-\mathrm{S}$ ) in the CGs is given in Table 4. According to Table 4 and Fig. $5 \mathrm{a}$ and $\mathrm{b}$, the $\mathrm{H}-\mathrm{S}$ method prevails in regions of the $\mathrm{B}$ group (arid and semi-arid) and the E group (polar/alpine/tundras), while the P-T method prevails in the regions of the A group (tropical/megathermal), the $\mathrm{C}$ group (temperate/mesothermal climates) and the D group (continental/microthermal). Even though the P-T method seems to be more powerful in more climatic zones, in reality the $\mathrm{H}-\mathrm{S}$ method prevails in $49.3 \%$ of the regions, while P-T does so
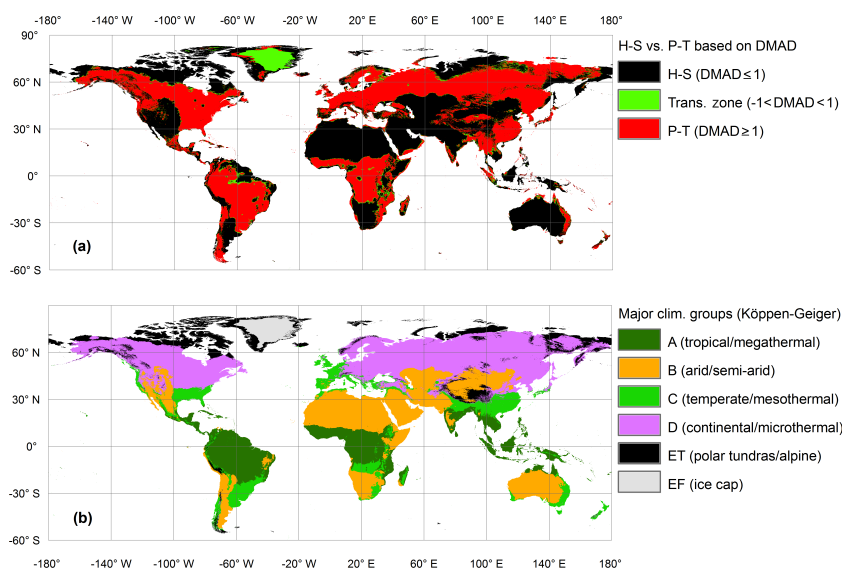

Figure 5. (a) Spatial extent of DMAD classes (P-T versus H-S) $\left(0.5^{\circ}\right.$ resolution map) and (b) spatial extent of the major climatic groups of the Köppen-Geiger climate classification according to Peel et al. (2007)

in $46.6 \%$ (the remaining proportion of $4.1 \%$ mainly corresponds to inner Greenland and very high mountain areas with annual $\mathrm{ET}_{\mathrm{O}}=0$ or to regions where both methods gave equal results). The better suitability of the standard $\mathrm{H}-\mathrm{S}$ method to drier environments and the respective suitability of the standard P-T method in more humid environments can be explained by the fact that the standard coefficient of $\mathrm{H}-\mathrm{S}$ was calibrated for Californian conditions (semi-arid/arid environments) (Hargreaves and Samani, 1985), while the standard coefficient of P-T was calibrated taking into account more humid environments (Priestley and Taylor, 1972).

The spatial variation in MAD \% for the case of $R_{\mathrm{S}}$ estimations using the standard solar radiation formula of $\mathrm{H}-\mathrm{S}$ for $K_{\mathrm{RS}}=0.17$ (Eq. 3) versus the mean annual $R_{\mathrm{S}}$ values of Sheffield et al. (2006) is given in Fig. 4d. It is indicative that $55.3 \%$ of the territories are included in the MAD \% range $\pm 10 \%$, while $95.2 \%$ is included in the range between $\pm 25 \%$ (Table 3 ). Significant deviations of $R_{\mathrm{s}}$ estimations using the standard H-S method appear mostly in the region of Greenland (Fig. 4d). The values of $R^{2}$ and RMSD (Table 3) indicate a good performance of the method in the case of monthly estimations. The overall results indicate that the use of the standard value $K_{\mathrm{RS}}=0.17$ can provide satisfactory indirect estimations of $R_{\mathrm{S}}$ for the greatest part of the world only by the use of temperature data.

\subsection{Partial weighted averages of mean monthly $a_{\mathrm{pt}}$, $c_{\mathrm{hs} 2}$ and $K_{\mathrm{RS}}$}

The PWAs of mean monthly $a_{\mathrm{pt}}$ and $c_{\mathrm{hs} 2}$ for short (PWA-s) and tall (PWA-t) reference crop were derived from the application of Eq. (7), and they are given in Fig. 6, while the respective PWAs of mean monthly $K_{\mathrm{RS}}$ values are given in Fig. 7. The global means of PWA of $a_{\mathrm{pt}}$ and $c_{\mathrm{hs} 2}$ for the short reference crop (presented below each map of Fig. 6a 
Table 4. Spatial extent of the major climatic groups CGs of the Köppen-Geiger climate map (Peel et al., 2007); spatial extent of DMAD classes (P-T versus H-S) within each CG.

\begin{tabular}{|c|c|c|c|c|}
\hline \multirow[t]{2}{*}{$\begin{array}{l}\text { CG of } \\
\text { Köppen-Geiger }\end{array}$} & \multirow[t]{2}{*}{$\begin{array}{r}\text { Percent coverage of CGs } \\
\text { based on Peel et al. (2007) map }\end{array}$} & \multicolumn{3}{|c|}{$\begin{array}{c}\text { Percent coverage of DMAD classes }{ }^{b} \\
\text { in a CG (P-T vs. H-S })\end{array}$} \\
\hline & & $\begin{array}{r}\mathrm{H}-\mathrm{S} \\
(\mathrm{DMAD} \leq-1)\end{array}$ & $\begin{array}{r}\text { Trans. zone } \\
-1<\text { DMAD }<1^{\mathrm{c}}\end{array}$ & $\begin{array}{r}\text { P-T } \\
(\mathrm{DMAD} \geq 1)\end{array}$ \\
\hline A - tropical/megathermal & $20.66 \%$ & $32.0 \%$ & $3.6 \%$ & $64.4 \%$ \\
\hline $\mathrm{B}-\operatorname{arid} / \mathrm{semi}$-arid & $32.90 \%$ & $86.4 \%$ & $1.3 \%$ & $12.3 \%$ \\
\hline $\mathrm{C}$ - temperate/mesothermal & $14.58 \%$ & $32.8 \%$ & $3.2 \%$ & $64.1 \%$ \\
\hline D - continental/microthermal & $27.00 \%$ & $26.9 \%$ & $2.1 \%$ & $71.0 \%$ \\
\hline E - polar/alpine (without Antarctica) & $4.86 \%$ & $71.1 \%$ & $16.3 \%{ }^{\mathrm{d}}$ & $12.5 \%$ \\
\hline
\end{tabular}

and c) and the global mean of PWA of $K_{\mathrm{RS}}$ values for $R_{\mathrm{S}}$ (presented below Fig. 7) approximate to the standard values of $a_{\mathrm{pt}}=1.26, c_{\mathrm{hs} 2}=0.0023$ and $K_{\mathrm{RS}}=0.17$, respectively.

As regards the spatial variation in $a_{\mathrm{pt}}$ for the short reference crop (Fig. 6a), the higher values were observed in extremely arid and desert environments exceeding the value of 1.8 (mainly due to extremely high vapour pressure deficit), while the extremely cold and extremely humid environments presented values $<1.0$. Interesting cases are the alpine tundra and extreme humid tropical environments, which presented similar values between $\sim 0.8-1.0$, due to the low values of the vapour pressure deficit. Values of $a_{\mathrm{pt}}$ below 0.8 were observed in subpolar areas. The spatial variation in $a_{\mathrm{pt}}$ for the tall reference crop (Fig. 6b) follows similar patterns to the $a_{\mathrm{pt}}$ of the short reference crop but with increased values, which can be described by the following relationship $a_{\mathrm{pt}(\mathrm{PWA}-\mathrm{t})}=1.73 \times a_{\mathrm{pt}(\mathrm{PWA}-\mathrm{s})}-0.58, R^{2}=0.996$, $p<0.0001$. This relationship is valid for $a_{\mathrm{pt}(\mathrm{PWA}-\mathrm{s})}>0.8$ for preserving $a_{\mathrm{pt}(\mathrm{PWA}-\mathrm{t})} \geq a_{\mathrm{pt}(\mathrm{PWA}-\mathrm{s})}$.

As regards the spatial variation in $c_{\mathrm{hs} 2}$ for the short reference crop (Fig. 6c), the higher values were observed in extremely arid and desert environments exceeding 0.0026 (mainly due to extremely high vapour pressure deficit) while the extremely cold and extremely humid environments presented values $<0.0018$. Similarities appear again in the case of alpine tundra and extreme humid tropical environments, which presented values between $\sim 0.0014$ and 0.0018 , due to the low values of the vapour pressure deficit. Values of $c_{\text {hs } 2}$ below 0.0014 were observed in subpolar areas. The spatial variation in $c_{\mathrm{hs} 2}$ for the tall reference crop (Fig. 6d) follows similar patterns with $c_{\mathrm{hs} 2}$ of the short reference crop but with increased values, which can be described by the following relationship $c_{\mathrm{hs} 2(\mathrm{PWA}-\mathrm{t})}=1.793 \times$ $c_{\text {hs2(PWA-s) }}-0.00114, R^{2}=0.967, p<0.0001$. This relationship is valid for $c_{\mathrm{hs} 2(\mathrm{PWA}-\mathrm{s})}>0.0014$ for preserving $c_{\mathrm{hs} 2(\mathrm{PWA}-\mathrm{t})} \geq c_{\mathrm{hs} 2(\mathrm{PWA}-\mathrm{s})}$.

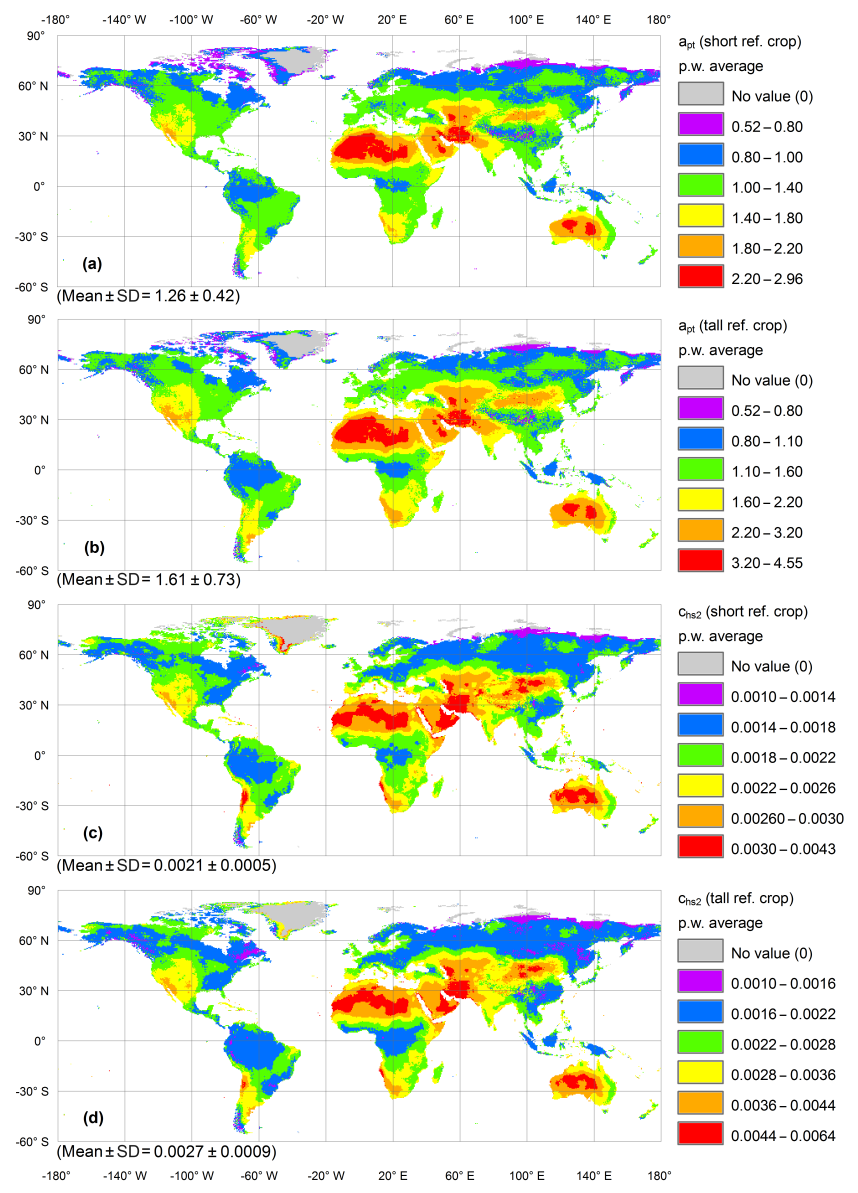

Figure 6. Partial weighted averages of mean monthly (a) $a_{\mathrm{pt}}$ for the short reference crop, (b) $a_{\mathrm{pt}}$ for the tall reference crop, (c) $c_{\mathrm{hs} 2}$ for the short reference crop and (d) $c_{\mathrm{hs} 2}$ for the tall reference crop $\left(0.5^{\circ}\right.$ resolution maps; mean $\pm \mathrm{SD}$ are estimated after conversion from WGS84 to Cylindrical Equal Area coordinate system excluding pixels of 0 value). 


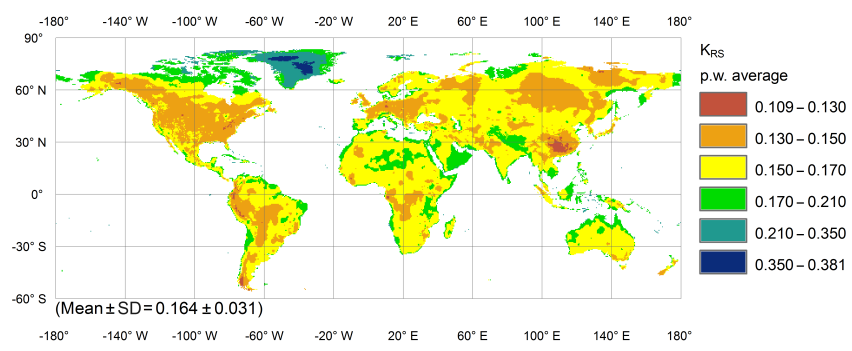

Figure 7. Partial weighted averages of mean monthly $K_{\mathrm{RS}}\left(0.5^{\circ}\right.$ resolution maps; mean $\pm \mathrm{SD}$ are estimated after conversion from WGS84 to Cylindrical Equal Area coordinate system).

In the case of $K_{\mathrm{RS}}$ (Fig. 7), extreme deviations from the value of 0.17 were observed in Greenland with values above 0.21 and in south-east China with values below 0.13 (regions of Chongqing, Guizhou, Hunan, Jiangxi and Guangxi). The spatial variation in $K_{\mathrm{RS}}$ does not follow a specific pattern in relation to climate zones, while in many cases, an increasing trend in its values closer to the coastlines was observed (Fig. 7). Additional observations about the effect of distance from the coastline (Dc) on $K_{\mathrm{RS}}$ are given in the Discussion section.

\subsection{Validation of the readjusted $a_{\mathrm{pt}}, c_{\mathrm{hs} 2}$ and $K_{\mathrm{RS}}$ coefficients}

The validation of the readjusted $a_{\mathrm{pt}}$ and $c_{\mathrm{hs} 2}$ coefficients for $\mathrm{ET}_{\mathrm{o}}$ estimations (for both reference crops) and the $K_{\mathrm{RS}}$ coefficient for $R_{\mathrm{S}}$ was performed taking into account the mean monthly values of the climatic parameters of all stations from Table 1. The readjusted coefficients for each station obtained from the $0.5^{\circ}$ resolution maps are given in Table S2 of the Supplement, while the comparison between $\mathrm{ET}_{\mathrm{o}}$ estimations (for both reference crops) and rasters and stations is provided in Fig. S2g and h, respectively. The comparison of different methods is described in the next paragraphs, while the overall results of the statistical criteria for all the examined cases are given in Table 5.

Table 5a and Fig. 8 show the $\mathrm{ET}_{\mathrm{o}}\left(\mathrm{mm} \mathrm{month}^{-1}\right)$ comparisons between the ASCE-short values versus the values of the $\mathrm{P}-\mathrm{T}$ and $\mathrm{H}-\mathrm{S}$ methods with the standard and the readjusted (PWA-s) coefficients and versus the values of the additional models given in Table 2. The following observations were derived from the results of Fig. 8 together with the results of the statistical criteria (Table 5a):

- The P-T(PWA-s) and H-S(PWA-s) models (Fig. 8b, d) outperformed the respective standard P-T(1.26) and $\mathrm{H}-$ $\mathrm{S}(0.0023)$ models (Fig. 8a, c) with regard to all the statistical criteria (Table 5a), reducing the RMSE values at 40 and $25 \%$, respectively.

- The comparison of statistical criteria between $\mathrm{H}$ S(0.0023), H-S(PWA-s), DRAL1 and DRAL2, which follow the general formula of the H-S method and are based on calibrations with global data, showed the following order of accuracy H-S(PWAs) $>$ DRAL1 $>$ DRAL2 $>$ H-S(0.0023).

- The standard P-T(1.26) showed the worst results for all criteria (Table 4a), while the use of P-T(PWA-s) succeeded in improving the predictions, giving better results for the H-S(0.0023), DRAL2, VAL1 and AKJ2 models.

- The H-S(PWA-s) provided better results for DRAL1, DRAL2, VAL1, AKJ1 and AKJ2 where the latter four require data for more climatic parameters.

- The order of accuracy of the models was the following: $\quad$ VAL3 $>$ VAL2 $>$ Copais $>$ H-S(PWA-s) $>$ AKJ1 $>$ P-T(PWA-s) $>$ DRAL1 $>$ DRAL2 $>$ H$\mathrm{S}(0.0023)>\mathrm{AKJ} 2>\mathrm{VAL} 1>\mathrm{P}-\mathrm{T}(1.26)$ (the order was based on absolute comparisons of the accuracy rankings for each criterion; see Table S3 in the Supplement). The RMSE difference between H-S(PWA-s) and the best VAL3 model was $6.8 \mathrm{~mm} \mathrm{month}^{-1}$ (or $0.23 \mathrm{~mm} \mathrm{~d}^{-1}$ ), while the respective difference between P-T(PWA-s) and VAL3 was $13.5 \mathrm{~mm} \mathrm{month}^{-1}$ (or $0.45 \mathrm{~mm} \mathrm{~d}^{-1}$ ). These differences are satisfactory, especially for the case of H-S(PWA-s), which uses fewer climatic data from VAL3. Of course, these differences are even smaller when compared to VAL2 and Copais, which also use more climatic parameters. Explanations of the less satisfactory performance of P-T(PWA-s) are given in the Discussion section.

Table 5b and Fig. 9a and b show the $\mathrm{ET}_{\mathrm{o}}\left(\mathrm{mm}_{\mathrm{month}}{ }^{-1}\right)$ comparisons between the ASCE-tall values versus the values of the P-T and H-S methods using the readjusted $a_{\mathrm{pt}}$ and $c_{\text {hs2 }}$ coefficients for tall reference crop (PWA-t), respectively. Since there are currently no other methods of reduced parameters calibrated based on $\mathrm{ASCE} \mathrm{ET}_{\mathrm{O}}$ for the tall reference crop, the comparison between the two methods is restricted. The results of Fig. 9a and $b$ together with the results of the statistical criteria (Table 5b) indicate a better performance of the H-S (with $c_{\text {hs } 2}=$ PWA-t). The higher errors observed in $\mathrm{H}-\mathrm{S}(\mathrm{PWA}-\mathrm{t})$ and P-T(PWA-t) in comparison to the respective errors of H-S(PWA-s) and P-T(PWA-s) for the short reference crop are explained by the fact that ASCE-tall is significantly higher than ASCE-short, especially in drier environments (ASCE-tall was found to be $\sim 28 \%$ higher than ASCE-short on a global scale based on the mean values given in Fig. $2 \mathrm{a}$ and $\mathrm{b}$, and $\sim 38 \%$ higher based on the comparison of the total mean values estimated by the Californian (USA) and Australian station data).

Table 5c and Fig. 10a and $\mathrm{b}$ show the comparisons between the $R_{\mathrm{S}}\left(\mathrm{MJ} \mathrm{m}^{-2} \mathrm{~d}^{-1}\right)$ of station data versus the respective values of the standard radiation formula of $\mathrm{H}-\mathrm{S}$ (Eq. 3) with $K_{\mathrm{RS}}=0.17$ and with $K_{\mathrm{RS}}=$ PWA, respectively. The results 

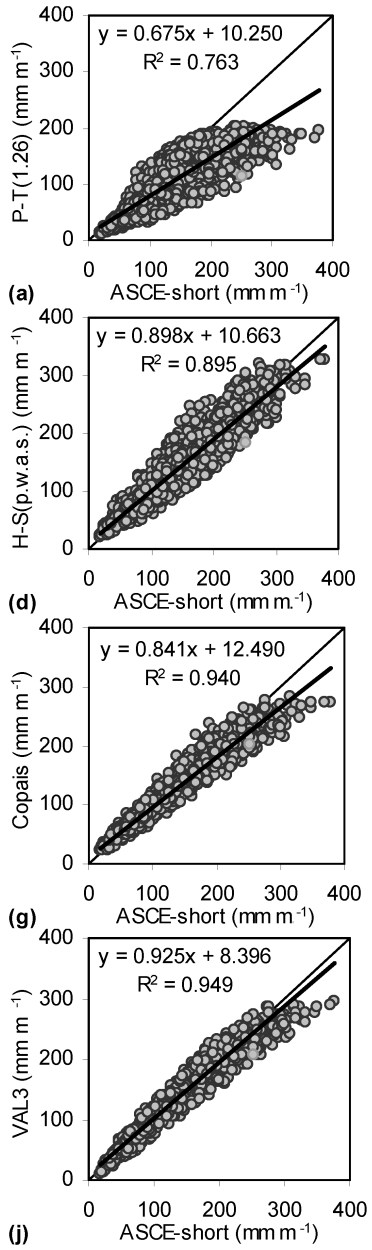
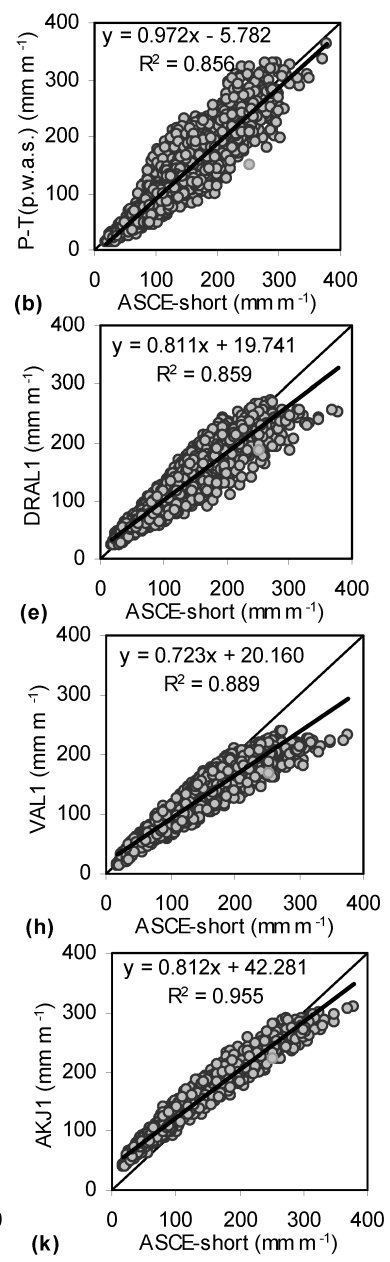
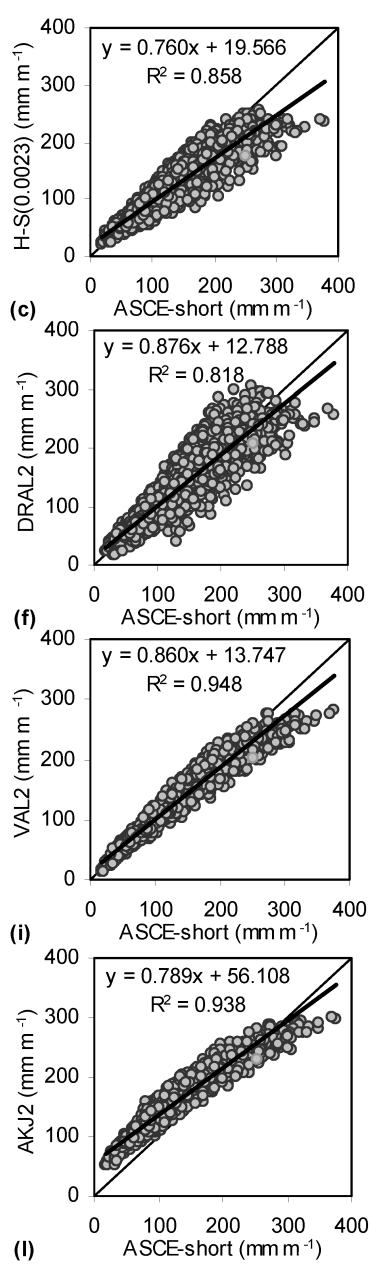

Figure 8. Comparative 1:1 plots between the results of $\mathrm{ET}_{\mathrm{o}}$ ASCE-short ( $\mathrm{mm}$ month ${ }^{-1}$ ) versus (a) the standard P-T method with $a_{\mathrm{pt}}=$ 1.26 , (b) the P-T method with $a_{\mathrm{pt}}=$ PWA-s $\left(0.5^{\circ}\right.$ resolution), (c) the standard H-S method with $c_{\mathrm{hs} 2}=0.0023$, (d) the H-S method with $c_{\mathrm{hs} 2}=$ PWA-s $\left(0.5^{\circ}\right.$ resolution), (e) DRAL1 (Eq. 8), (f) DRAL2 (Eq. 9), (g) Copais (Eq. 10), (h) VAL1 (Eq. 11), (i) VAL2 (Eq. 12), (j) VAL3 (Eq. 13), (k) AKJ1 (Eq. 14) and (l) AKJ2 (Eq. 15).

of Fig. 10a and b together with the results of the statistical criteria (Table 5c) indicate a better performance of the $\mathrm{H}$ $\mathrm{S} R_{\mathrm{S}}$ with $K_{\mathrm{RS}}=$ PWA even though the performance of the standard $\mathrm{H}-\mathrm{S} R_{\mathrm{S}}$ is also satisfactory.

\section{Discussion}

\subsection{Uncertainties in the data used for calibrating and validating the revised coefficients of the P-T and $\mathrm{H}$-S methods}

The recalibrated coefficients of the H-S and P-T methods were estimated using raster datasets that cover the period 1950-2000 assuming stationary climate conditions, while the validation datasets of Californian (USA) and Australian stations are expanded up to 2016. Recent studies have shown changes/anomalies after 2000 in temperature (Hansen et al., 2010; Sun et al., 2017), solar radiation (Wild et al., 2013), wind speed (McVicar et al., 2012a, b) and atmospheric humidity (Willet et al., 2014) and such changes could affect the validity of the revised coefficients. The comparisons of $T_{\max }$, $T_{\min }, R_{\mathrm{s}}, R_{\mathrm{n}}, \mathrm{DE}$ and $u_{2}$ values between the raster data and the station data showed a very good correspondence for the case of $T_{\max }, T_{\min }, R_{\mathrm{S}}$ and $R_{\mathrm{n}}$ (Fig. S2a, b, c, d) and a relatively good correspondence for the case of DE (Fig. S2e). In the case of $u_{2}$, a discrepancy was observed between the rasters and station data (Fig. S2f). The separate examination of $u_{2}$ for the USA-CA and Australian stations (Fig. S3) showed that the total average of mean monthly $u_{2}$ values of USA-CA stations was lower from the raster data of Sheffield et al. (2006) (data extracted from the stations' positions), while the opposite trend was found for the $u_{2}$ values of Australian stations. This discrepancy between the $u_{2}$ values of rasters and stations can be explained by the following: 
Table 5. Statistical criteria from the comparisons (a) between $\mathrm{ET}_{\mathrm{O}}$ values from ASCE-short and the methods used for estimating short reference crop evapotranspiration (i.e. P-T with standard and readjusted coefficients, H-S with standard and readjusted coefficients and all equations given in Table 2), (b) between ET $_{\mathrm{O}}$ values from ASCE-tall and P-T, H-S methods with readjusted coefficients for the tall reference crop, (c) $R_{\mathrm{S}}$ values from stations and $R_{\mathrm{S}}$ obtained from the $\mathrm{H}-\mathrm{S}$ radiation formula with standard and readjusted coefficients.

\begin{tabular}{|c|c|c|c|c|c|c|c|c|c|c|}
\hline Case & $\begin{array}{l}\text { Criterion } \\
\text { Optimum value }\end{array}$ & $\begin{array}{r}\text { MAE } \\
0\end{array}$ & $\begin{array}{r}\text { RMSE } \\
0\end{array}$ & $\begin{array}{r}\text { NRMSE \% } \\
0\end{array}$ & $\begin{array}{r}\text { PBIAS \% } \\
0\end{array}$ & $\begin{array}{r}R^{2} \\
\quad 1\end{array}$ & $b R^{2}$ & $\begin{array}{r}\mathrm{NSE} \\
1\end{array}$ & $\begin{array}{l}d \\
1\end{array}$ & $\begin{array}{r}\mathrm{KGE} \\
1\end{array}$ \\
\hline \multirow[t]{12}{*}{ (a) } & P-T (Eq. 2) with $a_{\mathrm{pt}}=1.26$ & 36.92 & 48.87 & 90.9 & 33.3 & 0.763 & 0.591 & 0.173 & 0.849 & 0.539 \\
\hline & P-T (Eq. 2) with $a_{\mathrm{pt}}=$ PWA-s & 22.71 & 29.43 & 40.3 & 7.5 & 0.856 & 0.832 & 0.837 & 0.956 & 0.883 \\
\hline & H-S (Eq. 4b) with $c_{\mathrm{hs} 2}=0.0023$ & 21.19 & 30.36 & 53.2 & 10.8 & 0.858 & 0.772 & 0.717 & 0.941 & 0.746 \\
\hline & H-S (Eq. $4 \mathrm{~b})$ with $c_{\mathrm{hs} 2}=$ PWA-s & 17.13 & 22.72 & 34.4 & 2.5 & 0.895 & 0.878 & 0.881 & 0.971 & 0.921 \\
\hline & DRAL1 (Eq. 8) & 19.53 & 27.05 & 44.5 & 4.8 & 0.859 & 0.818 & 0.802 & 0.955 & 0.833 \\
\hline & DRAL2 (Eq. 9) & 22.92 & 30.28 & 45.0 & 3.2 & 0.818 & 0.808 & 0.798 & 0.949 & 0.894 \\
\hline & Copais (Eq. 10) & 14.49 & 20.70 & 34.3 & 7.3 & 0.940 & 0.870 & 0.882 & 0.974 & 0.829 \\
\hline & VAL1 (Eq. 11) & 21.36 & 31.87 & 59.8 & 15.1 & 0.888 & 0.763 & 0.642 & 0.932 & 0.657 \\
\hline & VAL2 (Eq. 12) & 12.13 & 17.96 & 29.3 & 4.2 & 0.948 & 0.900 & 0.914 & 0.981 & 0.859 \\
\hline & VAL3 (Eq. 13) & 11.45 & 15.94 & 24.1 & 1.4 & 0.949 & 0.934 & 0.942 & 0.986 & 0.940 \\
\hline & AKJ1 (Eq. 14) & 21.17 & 24.24 & 42.0 & -10.6 & 0.955 & 0.887 & 0.824 & 0.964 & 0.771 \\
\hline & AKJ2 (Eq. 15) & 30.36 & 33.69 & 59.5 & -16.3 & 0.938 & 0.820 & 0.645 & 0.931 & 0.718 \\
\hline \multirow[t]{2}{*}{ (b) } & P-T (Eq. 2) with $a_{\mathrm{pt}}=$ PWA-t & 40.43 & 52.38 & 50.6 & 8.4 & 0.770 & 0.754 & 0.743 & 0.930 & 0.845 \\
\hline & H-S (Eq. 4b) with $c_{\mathrm{hs} 2}=$ PWA-t & 31.87 & 42.34 & 45.2 & 3.7 & 0.823 & 0.806 & 0.795 & 0.950 & 0.885 \\
\hline \multirow[t]{2}{*}{ (c) } & H-S $R_{\mathrm{S}}$ (Eq. 3) with $K_{\mathrm{RS}}=0.17$ & 1.64 & 1.99 & 29.6 & -4.5 & 0.930 & 0.885 & 0.912 & 0.977 & 0.932 \\
\hline & H-S $R_{\mathrm{S}}$ (Eq. 3) with $K_{\mathrm{RS}}=$ PWA & 1.05 & 1.43 & 22.3 & -0.8 & 0.952 & 0.944 & 0.950 & 0.988 & 0.972 \\
\hline
\end{tabular}

- Possible changes in wind speeds after 2000, since the majority of wind speed data in the station datasets corresponds to periods after 2000 .

- Uncertainties in the Sheffield et al. (2006) wind data due to the scarce existing wind data for calibrating their model on a global scale during the period of 1950-2000 and especially during the years belonging to the first half of the simulation period.

- The effect of the equation $u_{2}=4.87 u_{z} / \ln (67.8 z-5.42)$ (Allen et al., 1998, 2005), which was used to adjust the wind rasters of Sheffield et al. (2006) and the wind data of Australian stations from $z=10$ to $2 \mathrm{~m}$ height. The degree of accuracy of the aforementioned equation to convert wind data at $2 \mathrm{~m}$ is unknown. This equation is usually not calibrated for meteorological stations with anemometers positioned above $2 \mathrm{~m}$ height, while the uncertainty is even larger when it is applied on a global scale and for a pixel of $0.5^{\circ}$ resolution, which may contain high topographic variability.

- The bias that may have been introduced after cleaning extreme wind values in the data of USA-CA stations, which may be associated with hurricane events. The region of California is strongly affected by hurricanes and the higher wind speeds in the rasters of the Sheffield et al. (2006) data may partly have occurred because they included such events in their climatic simulations.
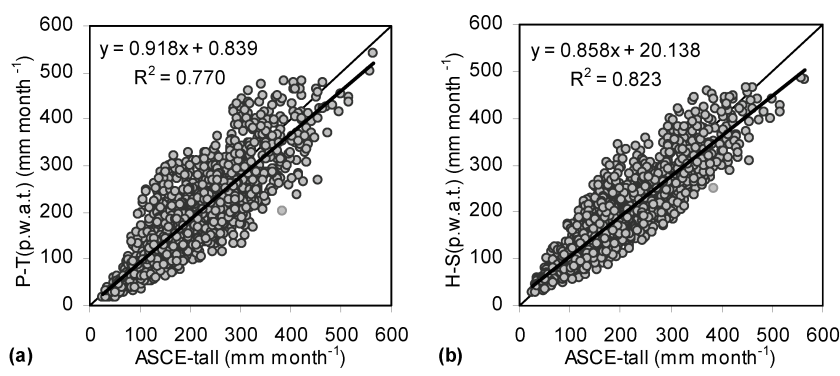

Figure 9. Comparative 1:1 plots between the results of $\mathrm{ET}_{\mathrm{O}}$ ASCE-tall (mm month ${ }^{-1}$ ) versus (a) the P-T method with $a_{\mathrm{pt}}=$ PWA-t $\left(0.5^{\circ}\right.$ resolution $)$ and (b) the H-S method with $c_{\text {hs } 2}=$ PWA-t $\left(0.5^{\circ}\right.$ resolution $)$.

- The bias that may have been introduced by the wind data of Australian stations. The AGBM database provides 12 values of mean monthly wind speeds of the total observation periods for 09:00 and another 12 values for 15:00 local time. In order to get the mean monthly wind speeds of the stations, the average value of 09:00 and 15:00 conditions was used for each month.

- Combinations of all the aforementioned cases.

Uncertainties may also exist in the case of $\mathrm{DE}=e_{\mathrm{s}}-e_{\mathrm{a}}$ (Fig. S2e), since Sheffield et al. (2006) provide data of specific humidity that were directly converted to actual vapour pressure $e_{\mathrm{a}}$ using the equation of Peixoto and Oort (1996), which uses the additional parameter of atmospheric pres- 

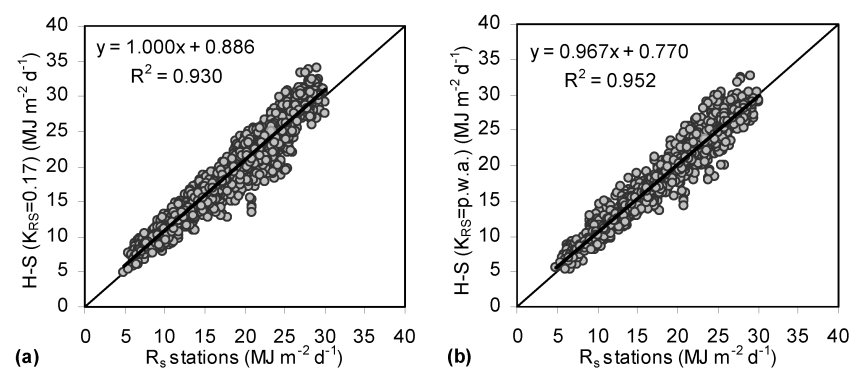

Figure 10. Comparative $1: 1$ plots between the $R_{\mathrm{S}}\left(\mathrm{MJ} \mathrm{m}^{-2} \mathrm{~d}^{-1}\right)$ values of USA-CA and Australian stations versus the results of $\mathrm{H}-\mathrm{S}$ radiation formula (Eq. 3) (a) with $K_{\mathrm{RS}}=0.17$ and (b) with $K_{\mathrm{RS}}=\mathrm{PWA}\left(0.5^{\circ}\right.$ resolution $)$.

sure as an internal parameter. The atmospheric pressure in the case of rasters was estimated based on elevation data of $1 \mathrm{~km}$ resolution ( $30 \mathrm{arcsec}$ ), which were further converted to $0.5^{\circ}$ resolution. The use of $e_{\mathrm{a}}$ data from $0.5^{\circ}$ resolution pixels may also have added additional error, especially when there is large topographic variability within the $0.5^{\circ}$ pixel. On the other hand, the $e_{\mathrm{a}}$ of stations was estimated by relative humidity and temperature data.

Thus, uncertainties exist in both rasters and station data. In future studies, further improvements in the revised coefficients can be made by using global raster data, which incorporate the conditions after 2000 , and by solving many of the aforementioned problems related to both station data and raster data produced by climatic models.

\subsection{Reasons for using annual PWA coefficients instead of monthly or seasonal ones in the case of $\mathrm{H}-\mathrm{S}$ and P-T methods}

The analysis presented in this study passed through various stages before the selection of the annual PWA form of the coefficients (Eq. 7). Some steps in the preliminary analysis were to analyse (a) the different forms of averages (e.g. mean, mode, median, geometric mean, harmonic mean) for deriving annual coefficients and (b) the strength of the derived mean monthly and seasonal coefficients versus the annual PWA coefficients and versus the coefficients of the standard methods,.

As regards the use of the weighted annual average (w.a.) of the mean monthly coefficients instead of other forms of averages (e.g. mean, mode, median, geometric mean - g.m., harmonic mean - h.m.), preliminary analysis was performed using data extracted by the climatic rasters from many positions in the world. During this analysis, trials to derive annual coefficients were made using an optimization algorithm separately for each position. The results showed that the optimized annual values were always closer to the monthly coefficients of the warmer months since the optimization algorithms try to reduce the total error, which is mainly dominated by the months that show larger $\mathrm{ET}_{\mathrm{o}}$ values (or $R_{\mathrm{S}}$ for the case of $K_{\mathrm{RS}}$ calibration). The optimized values were also compared to the different types of annual averages (e.g. mean, mode, median, g.m., h.m., w.a.), which were estimated after excluding values of monthly coefficients associated with months with $\mathrm{ET}_{\mathrm{o}}$ and $R_{\mathrm{S}}$ values $<45 \mathrm{~mm} \mathrm{month}^{-1}$ (for $R_{\mathrm{s}}$, the equivalent is $3.61 \mathrm{MJ} \mathrm{m}^{-2} \mathrm{~d}^{-1}$ ). The w.a. generally showed better performance in comparison to other types of annual averages because it is the only form that considers the amplitude of the parameter under investigation $\left(\mathrm{ET}_{\mathrm{O}}\right.$ and $R_{\mathrm{S}}$ ) (Eq. 7), giving more weight to the monthly coefficients that are related to the warmer months. This attribute of w.a. is extremely significant since it is the only type that considers the seasonal observed differences in monthly $\mathrm{ET}_{\mathrm{o}}$ (for $a_{\mathrm{pt}}$ and $c_{\mathrm{hs} 2}$ ) and $R_{\mathrm{s}}$ (for $K_{\mathrm{RS}}$ ) minimizing the possible errors during warmer months.

The case of mean monthly coefficients was also examined (results not shown). The results showed that the assessment of annual $\mathrm{ET}_{\mathrm{o}}$ and seasonal $\mathrm{ET}_{\mathrm{o}}$ during the warm season using the mean monthly coefficients is more accurate in comparison to the standard coefficients but not as good as the PWA coefficients, especially during the cold season. Similar findings were observed when different time intervals for calculating seasonal averages of the coefficients were used (e.g. 3-month averages or 6-month averages). The basic observed problem with monthly/seasonal coefficients associated with the global-scale application of this study was that many parts of the world presented unreasonably high or low monthly/seasonal values of the coefficients (at least 1 order of magnitude larger or smaller than the standard values) during cold seasons. This problem occurred because P-T and $\mathrm{H}-\mathrm{S}$ evapotranspiration models do not include the effect of humidity and wind, which becomes greater when temperature is low (in very low temperatures even the ASCE results can be questioned). Such values may lead to significant errors in monthly/seasonal $\mathrm{ET}_{\mathrm{o}}$ estimations during cold periods when there are deviations of climatic conditions (seasonal shifts/disturbances or climate changes in general) from those used for calibrating the coefficients. These were the reasons for using the threshold of $45 \mathrm{~mm} \mathrm{month}^{-1}$ to exclude such values from the PWA of the coefficients. Thus, the PWA annual values were chosen as the best solution for a global application because they counterbalance the errors that could be introduced by intra-annual/intra-seasonal climatic variability or other errors such as those described in the previous section of the Discussion section (errors associated with the data).

It is also important to note that the derivation of annual coefficients is a pure optimization problem when station data are used. For example, Cristea et al. (2013) derived coefficients of the P-T method for 106 stations that represent a range of climates across the contiguous USA. The coefficients were estimated for each station by minimizing the sum of the squared residuals between the benchmark FAO-56 and P-T using data only for the period April-September. The obtained optimized values of the coefficients were interpolated in order to make a map of the $a_{\mathrm{pt}}$ coefficient. In our study, the 
maps of the coefficients were produced based on raster data and not station data, which means that optimization should be performed pixel by pixel ( $\sim 62000$ pixels globally for the $0.5^{\circ}$ resolution excluding Antarctica). This procedure would require special programming since a readily available tool to perform this procedure does not exist in commercial or free GIS software packages. This is the main reason for using Eq. (7) as an alternative method in a GIS environment, since it can be calculated easily in raster calculators incorporated in the GIS packages. A solution could be the development of a tool for GIS purposes using raster data that is able to run using 24 rasters: 12 for the benchmark $\mathrm{ET}_{\mathrm{o}}$ and another 12 for the $\mathrm{P}-\mathrm{T}$ or $\mathrm{H}-\mathrm{S} \mathrm{ET}$ formula without the 1.26 and 0.0023 factors, respectively, in order to provide optimized annual values of their coefficients (for a global application, filters to remove unreasonable values are also required).

\subsection{Observations derived by the application of the $\mathrm{H}-\mathrm{S}$ radiation formula}

Special attention was also given in the case of $K_{\mathrm{RS}}$ coefficient for estimating $R_{\mathrm{S}}$. Although there were indications that the spatial variation in the PWA $a_{\mathrm{pt}}$ and $c_{\mathrm{hs} 2}$ coefficients on a global scale may be linked to general climatic characteristics (Fig. 5), the respective variation in PWA $K_{\mathrm{RS}}$ coefficient could not be clearly linked with a specific climatic or topographic characteristic. The only observed dependence, which showed some relevance to the spatial variation in $K_{\mathrm{RS}}$, was a relatively negative correlation with Dc. This observed dependence can be only used as a general observation and not as a basis for applying the empirical rule of Allen et al. (1998) in general $\left(K_{\mathrm{RS}}=0.16\right.$ for interior and $K_{\mathrm{RS}}=0.19$ for coastal locations). The large uncertainty in the aforementioned rule was also indicated by Samani (2000), and it is verified by the analysis presented in Fig. S4a of the Supplement. Figure $\mathrm{S} 4 \mathrm{a}$ shows a relatively negative correlation between $K_{\mathrm{RS}}$ and Dc (for Dc $<500 \mathrm{~km}$ ) but also shows an extremely high variability in $K_{\mathrm{RS}}$ close to the coastlines where $K_{\mathrm{RS}}$ values are not necessarily higher in comparison to the values observed in the interior regions. The observed lower variability in $K_{\mathrm{RS}}$ in interior regions is probably related to the fact that coastlines are more affected by oceanic climatic phenomena, which present high spatial variability on a global scale anyway. The raster data of $K_{\mathrm{RS}}$ (Fig. 7) can be used as indicator to control the validity of the rule but also to control the validity of the given values $0.16-0.19$ for a specific region. Samani (2000) also observed that the monthly $K_{\mathrm{RS}}$ values may be influenced by the difference between monthly maximum and minimum temperature, TD. This effect was also investigated through correlations between the mean monthly $K_{\mathrm{RS}}$ coefficients and the mean monthly TD values of the station data (Fig. S4b, in the Supplement). The results showed that the hypothesis related to the effect of TD on $K_{\mathrm{RS}}$ may be stronger locally in comparison to the effect of Dc, but again the variation in $K_{\mathrm{RS}}$ is extremely large in the TD range be- tween 8 and $15^{\circ} \mathrm{C}$ (Fig. S4b), not allowing secure conclusions for a global-scale application. The result of Fig. S4b is based only on the stations of Table 1 , and for this reason the variation on a global scale is expected to be much larger.

\subsection{Recommendations for reducing the uncertainties when the readjusted coefficients of the P-T and $\mathrm{H}-\mathrm{S}$ models are used}

The uncertainties, which may be introduced by climate disturbances/changes or other uncertainties related to the data used for calibrating the coefficients, can be reduced by taking into account some of the following observations and recommendations.

A separate analysis using only the stations of California showed that a regional mean value of the coefficients derived by PWA values may present even better performance because it probably counterbalances other uncertainties associated with the spatial climatic variability within a specific region. A factor for such uncertainties may be rainfall, which may not show significant seasonal deviations or deviations from the expected annual values for a large region but may show different spatial patterns every year within the region affecting the accuracy of the coefficients. The aforementioned observation was verified by the application of the $\mathrm{H}-\mathrm{S}$ method for $\mathrm{ET}_{\mathrm{o}}$ of the short reference crop for the stations of California when the average value of $c_{\mathrm{hs} 2}=0.0024$ obtained from the respective PWA-s values of the stations (Table S2) was used (this value also approximates the standard value of 0.0023 ). The average value of 60 PWA-s coefficients of the USA-CA stations gave better results for the individual coefficients (Fig. S5 and Table S4, in the Supplement). The aforementioned observations suggest that a robust territorial segmentation based on general topographic characteristics (e.g. elevation, slope, latitude and longitude, distance from the coastline) and general climatic characteristics (e.g. Köppen class, general precipitation and temperature patterns) can provide a proper zonation of large territories for deriving very robust mean values of $a_{\mathrm{pt}}, c_{\mathrm{hs} 2}$ and $K_{\mathrm{RS}}$ coefficients using the respective PWA values of each zone. Robust zonations based on grids of mean monthly precipitation and temperature using the data of Hijmans et al. (2005) or the mean monthly $\mathrm{ET}_{\mathrm{o}}$ rasters provided by this study can easily be performed using cluster analysis in a GIS environment (Demertzi et al., 2014; Aschonitis et al., 2016a, b).

The comparison between $\mathrm{P}-\mathrm{T}$ and $\mathrm{H}-\mathrm{S}$ evapotranspiration methods with readjusted coefficients but also their comparison with the other models in Table 2 also provided significant information. From the comparison between P-T and $\mathrm{H}-\mathrm{S}$ with readjusted coefficients, it was observed that $\mathrm{H}-\mathrm{S}$ provided better results in both the short and the tall reference crop. The better performance of $\mathrm{H}-\mathrm{S}$ can be attributed to the fact that more than $\sim 80 \%$ of stations from Table 1 are located in territories with negative DMAD values (Fig. 5a) giving a general advantage to the $\mathrm{H}-\mathrm{S}$ method for more robust 
estimations. This observation can explain the better performance of the standard H-S (with $c_{\mathrm{hs} 2}=0.0023$ ) in comparison to the standard P-T (with $a_{\mathrm{pt}}=1.26$ ) for the short reference crop (Table 5a) and indirectly validates the DMAD map. Considering these observations, it is recommended to take into account both the MAD (Fig. 4,b,c) and DMAD (Fig. 5a) maps before selecting one of the two methods either using the standard or the PWA coefficients. From the comparisons with the other models in Table 2, it was observed that three models, which use temperature, radiation and humidity data (i.e. VAL3, VAL2, Copais and especially VAL3), provided better estimations. These models have shown very good performance using data from other case studies (Pan et al., 2011; Shiri et al., 2014; Kisi, 2014; Gao et al., 2015; Valipour, 2015a, c; Djaman et al., 2015, 2016, 2017; Ahooghalandari et al., 2017), and their use is recommended instead of the P-T and $\mathrm{H}-\mathrm{S}$ with readjusted coefficients when the only missing climatic parameter is wind speed.

A very interesting observation was also made about the tall reference crop based on the results of the MAD \% map (Fig. $4 a$ ). In the MAD \% class of $\pm 10 \%$ of Fig. $4 a$, some small negative values were observed, which correspond to $\sim 2 \%$ of map coverage. These values indicate slightly larger annual values of ASCE-short in comparison to ASCE-tall. This result was observed in regions of extremely small vapour pressure deficit (areas of very high elevation, either of very cold or extremely humid conditions scattered around the world), and it is a peculiarity of Eq. (1) and probably an artefact. This result occurred because the second term of the nominator in Eq. (1) (which includes the vapour pressure deficit term and the $C_{\mathrm{n}}$ coefficient) approximates to 0 when $e_{\mathrm{s}}-e_{\mathrm{a}}$ becomes extremely small, while the denominator of Eq. (1) is always larger in ASCE-tall in comparison to ASCE-short due to the difference in $C_{\mathrm{d}}$ value ( 0.34 for the short and 0.38 for the tall reference crop). A recommendation for partly solving this problem for the tall reference crop applications is to use the revised coefficients of the P-T and H-S methods derived for the short reference crop in the places were the annual value of ASCE-tall is lower than ASCEshort. This recommendation is based on the fact that annual ASCE-tall is expected to be always larger than the respective value of ASCE-short. This peculiarity was not corrected in the ASCE-tall maps and the respective $a_{\mathrm{pt}}$ and $c_{\mathrm{hs} 2}$ coefficients for the tall reference crop in order to show the absolute estimations of ASCE-tall and the respective coefficients. Finding the negative values in the MAD \% map (Fig. 4a), the users can identify the location of these pixels.

\section{Data availability}

The datasets produced in this study have been archived in the PANGAEA database (https://doi.org/10.1594/PANGAEA.868808) and in the ESRN database, which is currently supported by the Univer- sity of Ferrara (Italy), Aristotle University of Thessaloniki (Greece) and the University of Campania Luigi Vanvitelli (Italy) (http://www.esrn-database.org/gis-data.html or http://esrn-database.weebly.com/gis-data.html). Apart from the $0.5^{\circ}$ resolution raster datasets, the database contains the same datasets at finer resolution $(30 \mathrm{arcsec}, 2.5 \mathrm{arcmin}$, 5 arcmin and 10 arcmin). These finer datasets are provided in order to cover the observed resolution range in the initial climatic data (e.g. the temperature data of Hijmans et al. (2005) are provided at $30 \mathrm{arcsec}$ resolution). The finer resolutions were produced using bilinear interpolation on solar radiation, humidity and wind speed data of Sheffield et al. (2006). This interpolation method is not the most appropriate for such purposes. The data of finer resolutions can only be used as a tool to assess uncertainties associated with temperature variation effects within a $0.5^{\circ}$ pixel or to estimate average values of the coefficients for larger territories in order to capture a better representation of the coastlines or islands that do not exist at $0.5^{\circ}$ resolution (use of values from individual pixels is not recommended). A complete list of the datasets is provided in the Table S5.

\section{Conclusions}

The study provided global grids of revised annual coefficients for the P-T and H-S methods for estimating $\mathrm{ET}_{\mathrm{o}}$ for both short and tall reference crops. The coefficients were calibrated using respective grids of $\mathrm{ET}_{\mathrm{O}}$ estimated with the ASCE-standardized method. Respective grids of annual coefficients were also derived for the radiation formula of $\mathrm{H}-$ $\mathrm{S}$. The calibration procedures were based on global gridded climatic data of the period 1950-2000. The method for deriving annual coefficients of the P-T and H-S methods was based on partial weighted averages (PWAs) of the respective mean monthly coefficients. This method estimates the annual values considering the amplitude of the parameter under investigation $\left(\mathrm{ET}_{\mathrm{o}}\right.$ and $R_{\mathrm{S}}$ ) giving more weight to the monthly coefficients of the months with higher $\mathrm{ET}_{\mathrm{o}}$ values (or $R_{\mathrm{S}}$ values for the case of the $\mathrm{H}-\mathrm{S}$ radiation formula). The method also eliminates the effect of unreasonable monthly coefficients that may occur during periods when $\mathrm{ET}_{\mathrm{o}}$ and $R_{\mathrm{S}}$ fall below a specific threshold. The new coefficients were validated based on data from 140 stations located at various climatic zones of the USA and Australia with expanded observations up to 2016. Additional tests were also performed for the case of the short reference crop evapotranspiration using additional models with low requirements for climatic data. The validation procedure for $\mathrm{ET}_{\mathrm{o}}$ estimations of the short reference crop showed that the P-T and H-S methods with revised coefficients outperformed the standard methods reducing the estimated RMSE in $\mathrm{ET}_{\mathrm{o}}$ values by 40 and $25 \%$, respectively. The estimations of $R_{\mathrm{S}}$ using the $\mathrm{H}-\mathrm{S}$ formula with revised coefficients reduced the RMSE by $28 \%$ in comparison to the standard formula. The comparisons with 
other models of the short reference crop showed that the P-T and $\mathrm{H}-\mathrm{S}$ methods with revised coefficients can compete with models of additional climatic parameters. In the case where only wind speed is missing from available data, the use of the VAL2, VAL3 and Copais methods (temperature, radiation and humidity data requirements) is recommended. Finally, a raster database of $0.5^{\circ}$ resolution was built consisting of (a) global maps for the mean monthly $\mathrm{ET}_{\mathrm{o}}$ values estimated by the ASCE-standardized method for both reference crops, (b) global maps for the revised annual coefficients of the P-T and $\mathrm{H}-\mathrm{S}$ evapotranspiration methods for both reference crops and a global map for the revised annual coefficients of the $\mathrm{H}-\mathrm{S}$ radiation formula, and (c) global maps that indicate the optimum locations for using the standard P-T and H-S methods and their possible annual errors based on reference values (MAD \% and DMAD maps). The online free availability of the database can support estimations of $\mathrm{ET}_{\mathrm{o}}$ and solar radiation for locations where climatic data are limited, and it can support studies which require such estimations on larger scales (e.g. country, continent, world).

The methods used in this study, their respective results and the observed uncertainties can be used as a base for future work focusing on

a. the validation of the coefficients for other parts of the world, especially using climatic data obtained after 2000 , and the comparison with other models of low data requirements

b. the recalibration of the coefficients using data from climatic models that include observations from more recent years and an analysis of climate change effects on the coefficients

c. the use of the available climatic datasets obtained from climatic models in order to calibrate models of the coefficients for various locations providing values that are not fixed, such as the ones given in this study,

$\mathrm{d}$ analysis of alternative methods for deriving annual coefficients that approximate optimized values or the incorporation of optimization algorithms in a GIS environment to capture the optimum solution per pixel

e. the confrontation of uncertainties related to the data used for calibration and validation (e.g. low representativity of interpolated climatic parameters due to the lack of data in many parts of the world; errors associated with commonly used equations, such as the one used for adjusting wind data at $2 \mathrm{~m}$ height; uncertainties associated with the observed data).

\section{The Supplement related to this article is available online at https://doi.org/10.5194/essd-9-615-2017-supplement.}

Competing interests. The authors declare that they have no conflict of interest.

Acknowledgements. This study was performed in the context of two post-doctoral research studies by Vassilis Aschonitis financed by Ferrara University (Italy) and Aristotle University of Thessaloniki (Greece).

Edited by: David Carlson

Reviewed by: two anonymous referees

\section{References}

Abtew, W.: Evapotranspiration measurements and methoding for three wetland systems in South Florida, J. Am. Water Resour. As., 32, 465-473, 1996.

Ahooghalandari, M., Khiadani, M., and Jahromi, M. E.: Developing Equations for Estimating Reference Evapotranspiration in Australia, Water Resour. M., 30, 3815-3828, 2016.

Ahooghalandari, M., Khiadani, M., and Jahromi, M. E.: Calibration of Valiantzas' reference evapotranspiration equations for the Pilbara region, Western Australia, Theor. Appl. Climatol., 128, 845-856, 2017.

Allen, R. G., Pereira, L. S., Raes, D., and Smith, M.: Crop Evapotranspiration: Guidelines for computing crop water requirements, Irrigation and Drainage Paper 56, Food and Agriculture Organization of the United Nations, Rome, 1998.

Allen, R. G., Walter, I. A., Elliott, R., Howell, T., Itenfisu, D., and Jensen M.: The ASCE standardized reference evapotranspiration equation. Final Report (ASCE-EWRI) Pr., in: Environmental and Water Resources Institute, 2005, edited by: Allen, R. G., Walter, I. A., Elliott, R., Howell, T., Itenfisu, D., and Jensen, M., Task Committee on Standardization of Reference Evapotranspiration of the Environmental and Water Resources Institute, 2005.

Alexandris, S., Kerkides, P., and Liakatas, A.: Daily reference evapotranspiration estimates by the "Copais" approach, Agr. Water Manage., 82, 371-386, 2006.

Aschonitis, V., Antonopoulos, V. Z., and Papamichail, D. M.: Evaluation of pan coefficient equations in a semi-arid Mediterranean environment using the ASCE standardized Penman-Monteith method, Agr. Sci., 3, 58-65, 2012.

Aschonitis, V., Demertzi, K., Papamichail, D., Colombani, N., and Mastrocicco, M.: Revisiting the Priestley-Taylor method for the assessment of reference crop evapotranspiration in Italy, Ital. J. Agrometeorol., 20, 5-18, 2015.

Aschonitis, V., Miliaresis, G., Demertzi, K., and Papamichail, D.: Terrain segmentation of Greece using the spatial and seasonal variation of reference crop evapotranspiratiom, Adv. Meteorol., 3092671, 1-14, https://doi.org/10.1155/2016/3092671, 2016 .

Aschonitis, V., Awe, G. O., Abegunrin, T. P., Demertzi, K. A., Papamichail, D. M., and Castaldelli, G.: Geographic segmentation, spatial dependencies, and evaluation of the relative position of rain-gauge based on gridded data of mean monthly precipitation: application in Nigeria, Hydrol. Res., nh2016095, https://doi.org/10.2166/nh.2016.095, in press, 2016 b. 
Azhar, A. H. and Perera, B. J. C.: Evaluation of reference evapotranspiration estimation methods under Southeast Australian conditions, J. Irrig. Drain E.-ASCE, 137, 268-279, 2011.

Bachour, R., Walker, W. R., Torres-Rua, A. F., and McKee, M.: Assessment of reference evapotranspiration by the hargreaves method in the Bekaa Valley, Lebanon, J. Irrig. Drain. E.-ASCE, 139, 933-938, 2013.

Berti, A., Tardivo, G., Chiaudani, A., Rech, F., and Borin, M.: Assessing reference evapotranspiration by the Hargreaves method in north-eastern Italy, Agr. Water Manage., 140, 20-25, 2014.

Brinckmann, S., Krähenmann, S., and Bissolli, P.: Highresolution daily gridded data sets of air temperature and wind speed for Europe, Earth Syst. Sci. Data, 8, 491-516, https://doi.org/10.5194/essd-8-491-2016, 2016.

Castellvi, F., Stockle, C. O., Perez, P. J., and Ibanez, M.: Comparison of methods for applying the Priestley-Taylor equation at a regional scale, Hydrol. Process., 15, 1609-1620, 2001.

Cristea, N. C., Kampf, S. K., and Burges, S. J.: Revised coefficients for Priestley-Taylor and Makkink-Hansen equations for estimating daily reference evapotranspiration, J. Hydrol. Eng., 18, 12891300, 2013.

Demertzi, K., Papamichail, D., Aschonitis, V., and Miliaresis, G.: Spatial and seasonal patterns of precipitation in Greece: The terrain segmentation approach, Global Nest J., 16, 988-997, 2014.

Djaman, K., Balde, A. B., Sow, A., Muller, B., Irmak, S., N'Diaye, M. K., Manneh, B., Moukoumbi, Y. D., Futakuchi, K., and Saito, K.: Evaluation of sixteen reference evapotranspiration methods under sahelian conditions in the Senegal River Valley, J. Hydrol. Reg. Stud., 3, 139-159, 2015.

Djaman, K., Irmak, S., Kabenge, I., and Futakuchi, K.: Evaluation of FAO-56 penman-monteith model with limited data and the valiantzas models for estimating grass-reference evapotranspiration in Sahelian conditions, J. Irrig. Drain. E.ASCE, 142, 04016044, https://doi.org/10.1061/(ASCE)IR.19434774.0001070, 2016.

Djaman, K., Irmak, S., and Futakuchi, K.: Daily reference evapotranspiration estimation under limited data in eastern Africa, J. Irrig. Drain E.-ASCE, 143, 06016015, https://doi.org/10.1061/(ASCE)IR.1943-4774.0001154, 2017.

Droogers, P. and Allen, R. G.: Estimating reference evapotranspiration under inaccurate data conditions, Irrig. Drain. Syst., 16, 33-45, 2002.

Eichinger, W. E., Parlange, M. B., and Strickler, H.: On the concept of equilibrium evaporation and the value of the Priestley-Taylor coefficient, Water Resour. Res., 32, 161-164, 1996.

Flint, A. L. and Childs, S. W.: Use of the Priestley-Taylor evaporation equation for soil water limited conditions in a small forest clearcut, Agr. Forest Meteorol., 56, 247-260, 1991.

Gao, X., Peng, S., Xu, J., Yang, S., and Wang, W.: Proper methods and its calibration for estimating reference evapotranspiration using limited climatic data in Southwestern China, Arch. Agron. Soil Sci., 61, 415-426, 2015.

Giles, D. G., Black, T. A., and Spittlehouse, D. L.: Determination of growing season soil water deficits on a forested slope using water balance analysis, Can. J. Forest Res., 15, 107-114, 1984.

Gupta, H. V., Kling, H., Yilmaz, K. K., and Martinez, G. F.: Decomposition of the mean squared error and NSE performance criteria: Implications for improving hydrological modelling, J. Hydrol., 377, 80-91, 2009.
Hansen, J., Ruedy, R., Sato, M., and Lo K.: Global surface temperature change, Rev. Geophys., 48, RG4004, https://doi.org/10.1029/2010RG000345, 2010.

Hargreaves, G. H. and Allen, R. G.: History and evaluation of hargreaves evapotranspiration equation, J. Irrig. Drain E.-ASCE, 129, 53-63, 2002.

Hargreaves, G. H. and Samani, Z. A.: Estimating potential evapotranspiration, J. Irrig. Drain E.-ASCE, 108, 223-230, 1982.

Hargreaves, G. H. and Samani, Z. A.: Reference crop evapotranspiration from ambient air temperature, American Society of Agricultural Engineers, 12 pp., availabe at: http://libcatalog.cimmyt. org/download/reprints/97977.pdf (last access: 1 October 2016), 1985.

Heydari, M. M. and Heydari, M.: Calibration of Hargreaves-Samani equation for estimating reference evapotranspiration in semiarid and arid regions, Arch. Agron. Soil Sci., 60, 695-713, 2014.

Hijmans, R. J., Cameron, S. E., Parra, J. L., Jones, P. G., and Jarvis, A.: Very high resolution interpolated climate surfaces for global land areas, Int. J. Climatol., 25, 1965-1978, 2005.

Itenfisu, D., Elliott, R. L., Allen, R. G., and Walter, I. A.: Comparison of reference evapotranspiration calculations as part of the ASCE standardization effort, J. Irrig. Drain E.-ASCE, 129, 440448, 2003.

Kellner, E.: Surface energy fluxes and control of evapotranspiration from a Swedish Sphagnum mire, Agr. Forest Meteorol., 110, 101-123, 2001.

Kisi, O.: Comparison of different empirical methods for estimating daily reference evapotranspiration in mediterranean climate, J. Irrig. Drain E.-ASCE, 140, 04013002, https://doi.org/10.1061/(ASCE)IR.1943-4774.0000664, 2014.

Krause, P., Boyle, D. P., and Bäse, F.: Comparison of different efficiency criteria for hydrological model assessment, Adv. Geosci., 5, 89-97, https://doi.org/10.5194/adgeo-5-89-2005, 2005.

Lhomme, J. -P.: A theoretical basis for the Priestley-Taylor coefficient, Bound.-Lay. Meteorol., 82, 179-191, 1997.

Long, H., Shuai, X., Lei, Q., and Zhang, R.: Spatiotemporal distribution of calibration coefficients of hargreaves equation for estimating potential evapotranspiration in Mainland China, J. Irrig. Drain E.-ASCE, 139, 293-299, 2013.

Maidment, D. R.: Handbook of hydrology, McGraw-Hill, New York, 1992.

McMahon, T. A., Peel, M. C., Lowe, L., Srikanthan, R., and McVicar, T. R.: Estimating actual, potential, reference crop and pan evaporation using standard meteorological data: a pragmatic synthesis, Hydrol. Earth Syst. Sci., 17, 1331-1363, https://doi.org/10.5194/hess-17-1331-2013, 2013.

McVicar, T. R., Roderick, M. L., Donohue, R. J., and Van Niel, T. G.: Less bluster ahead? ecohydrological implications of global trends of terrestrial near-surface wind speeds, Ecohydrology, 5, 381-388, 2012a.

McVicar, T. R., Roderick, M. L., Donohue, R. J., Li, L. T., Van Niel, T. G., Thomas, A., Grieser, J., Jhajharia, D., Himri, Y., Mahowald, N. M., Mescherskaya, A. V., Kruger, A. C., Rehman, S., and Dinpashoh, Y.: Global review and synthesis of trends in observed terrestrial near-surface wind speeds: implications for evaporation, J. Hydrol., 416-417, 182-205, 2012b.

Mendicino, G. and Senatore, A.: Regionalization of the hargreaves coefficient for the assessment of distributed reference evapotran- 
spiration in Southern Italy, J. Irrig. Drain. E.-ASCE, 139, 349$362,2013$.

Mintz, Y. and Walker, G. K.: Global fields of soil moisture and land surface evapotranspiration derived from observed precipitation and surface air temperature, J. Appl. Meteorol., 32, 1305-1334, 1993.

Moges, S. A., Katambara, Z., and Bashar, K.: Decision support system for estimation of potential evapotranspiration in Pangani Basin, Phys. Chem. Earth, 28, 927-934, 2003.

Mohawesh, O. E. and Talozi, S. A.: Comparison of Hargreaves and FAO56 equations for estimating monthly evapotranspiration for semi-arid and arid environments, Arch. Agron. Soil Sci., 58, 321-334, 2012.

Nash, J. E. and Sutcliffe, J. V.: River flow forecasting through conceptual models, Part I - A discussion of principles, J Hydrol., 10, 282-290, 1970.

Ngongondo, C., Xu, C. -Y., Tallaksen, L. M., and Alemaw, B.: Evaluation of the FAO Penman-montheith, Priestley-Taylor and Hargreaves models for estimating reference evapotranspiration in southern Malawi, Hydrol. Res., 44, 706-722, 2013.

Osborn, T. J. and Jones, P. D.: The CRUTEM4 land-surface air temperature data set: construction, previous versions and dissemination via Google Earth, Earth Syst. Sci. Data, 6, 61-68, https://doi.org/10.5194/essd-6-61-2014, 2014.

Pan, Y., Gong, H.-L., Li, X.-J., Zhu, L., and Zhang, J.: Application of Valiantzas approach to estimating reference evapotranspiration in China. Shuikexue Jinzhan, Adv. Water Sci., 22, 30-37, 2011.

Peel, M. C., Finlayson, B. L., and McMahon, T. A.: Updated world map of the Köppen-Geiger climate classification, Hydrol. Earth Syst. Sci., 11, 1633-1644, https://doi.org/10.5194/hess-11-16332007, 2007.

Peixoto, J. P. and Oort, A. H.: The climatology of relative humidity in the atmosphere, J. Climate, 9, 3443-3463, 1996.

Pereira, A. R.: The Priestley-Taylor parameter and the decoupling factor for estimating reference evapotranspiration, Agr. Forest Meteorol., 125, 305-313, 2004.

Peterson, T. C. and Vose, R. S.: An overview of the global historical climatology network temperature database, B. Am. Meteorol. Soc., 78, 2837-2849, 1997.

Priestley, C. H. B. and Taylor, R. J.: On the assessment of surface heat flux and evaporation using large-scale parameters, Mon. Weather Rev., 100, 81-92, 1972.

Rahimikhoob, A., Behbahani, M. R., and Fakheri, J.: An evaluation of four reference evapotranspiration models in a subtropical climate, Water Resour. Manage., 26, 2867-2881, 2012.

Ravazzani, G., Corbari, C., Morella, S., Gianol, P., and Mancini, M.: Modified Hargreaves-Samani equation for the assessment of reference evapotranspiration in alpine river basins, J. Irrig. Drain. E.-ASCE, 138, 592-599, 2012.

Samani, Z.: Estimating solar radiation and evapotranspiration using minimum climatological data, J. Irrig. Drain. E.-ASCE, 126, 265-267, 2000.

Sheffield, J., Goteti, G., and Wood, E. F.: Development of a 50-yr high-resolution global dataset of meteorological forcings for land surface modeling, J. Climate, 19, 3088-3111, 2006.

Shiri, J., Nazemi, A. H., Sadraddini, A. A., Landeras, G., Kisi, O., Fakheri Fard, A., and Marti, P.: Comparison of heuristic and empirical approaches for estimating reference evapotranspiration from limited inputs in Iran, Comput. Electron. Agr., 108, 230241, 2014.

Shuttleworth, W. J. and Calder, I. R.: Has the Priestley-Taylor equation any relevance to forest evaporation?, J. Appl. Meteorol., 18, 639-646, 1979.

Singh, R. K. and Irmak, A.: Treatment of anchor pixels in the METRIC model for improved estimation of sensible and latent heat fluxes, Hydrol. Sci. J., 56, 895-906, 2011.

Snyder, R. L. and Pruitt. W. O.: Estimating reference evapotranspiration with hourly data, VII-1-VII-3, edited by: Snyder, R., Henderson, D. W., Pruitt, W. O., and Dong, A.Calif. Irrig. Mgmt. Systems, Final Rep., Univ. Calif., Davis, 1985.

Snyder, R. L. and Pruitt. W. O.: Evapotranspiration data management in California, presented at the Amer. Soc. of Civil Engr. Water Forum “92”, 2-6 August 1992, Baltimore, MD, 1992.

Sumner, D. M. and Jacobs, J. M.: Utility of Penman-Monteith, Priestley-Taylor, reference evapotranspiration, and pan evaporation methods to estimate pasture evapotranspiration, J. Hydrol., 308, 81-104, 2005.

Sun, X., Ren, G., Xu, W., Li, Q., and Ren, Y.: Global land-surface air temperature change based on the new CMA GLSAT data set, Sci. Bull., 62, 236-238, 2017.

Tabari, H.: Evaluation of reference crop evapotranspiration equations in various climates, Water Resour. Manage., 24, 23112337, 2010.

Tabari, H. and Talaee, P. H.: Local calibration of the Hargreaves and Priestley-Taylor equations for estimating reference evapotranspiration in arid and cold climates of Iran based on the PenmanMonteith model, J. Hydrol. Eng., 16, 837-845, 2011.

Tateishi, R. and Ahn, C. H.: Mapping evapotranspiration and water balance for global land surfaces, ISPRS J. Photogramm., 51, 209-215, 1996.

Thornthwaite, C. W.: An approach toward a rational classification of climate, Geogr. Rev., 38, 55-94, 1948.

Trajkovic, S.: Hargreaves versus Penman-Monteith under humid condition, J. Irrig. Drain. E.-ASCE, 133, 38-42, 2007.

Valiantzas, J. D.: Simple ETo forms of Penman's equation without wind and/or humidity data. I: Theoretical development, J. Irrig. Drain E.-ASCE, 139, 1-8, 2013a.

Valiantzas, J. D.: Simplified reference evapotranspiration formula using an empirical impact factor for penman's aerodynamic term, J. Hydrol. Eng., 18, 108-114, 2013 b.

Valiantzas, J. D.: Simple ETo forms of Penman's equation without wind and/or humidity data. II: Comparisons with reduced setFAO and other methodologies, J. Irrig. Drain. E.-ASCE, 139, 9$19,2013 \mathrm{c}$.

Valiantzas, J. D.: Closure to "Simple ETo forms of Penman's equation without wind and/or humidity data. I: Theoretical development" by John D. Valiantzas, J. Irrig. Drain E.ASCE, 140, 07014017, https://doi.org/10.1061/(ASCE)IR.19434774.0000750, 2014.

Valipour, M.: Ability of Box-Jenkins models to estimate of reference potential evapotranspiration (A Case Study: Mehrabad synoptic station, Tehran, Iran), IOSR J. Agr. Vet. Sci., 1, 1-11, 2012.

Valipour, M.: Application of new mass transfer formulae for computation of evapotranspiration, J. Appl. Water Eng. Res., 2, 33-46, 2014.

Valipour, M.: Investigation of Valiantzas' evapotranspiration equation in Iran, Theor. Appl. Climatol., 121, 267-278, 2015a. 
Valipour, M.: Evaluation of radiation methods to study potential evapotranspiration of 31 provinces, Meteorol. Atmos. Phys., 127, 289-303, 2015b.

Valipour, M.: Importance of solar radiation, temperature, relative humidity, and wind speed for calculation of reference evapotranspiration, Arch. Agron. Soil Sci., 61, 239-255, 2015c.

Valipour, M.: Analysis of potential evapotranspiration using limited weather data, Appl. Water Sci., 7, 187-197, 2017.

Valipour, M. and Gholami Sefidkouhi, M. A.: Temporal analysis of reference evapotranspiration to detect variation factors, Int. J. Global Warm., https://doi.org/10.1504/IJGW.2018.10002058, in press, 2017.

Valipour, M., Gholami Sefidkouhi, M. A., and Raeini-Sarjaz, M.: Selecting the best model to estimate potential evapotranspiration with respect to climate change and magnitudes of extreme events, Agr. Water Manage., 180, 50-60, 2017.

Wang, K. and Dickinson, R. E.: A review of global terrestrial evapotranspiration: Observation, modeling, climatology, and climatic variability, Rev. Geophys., 50, RG2005, https://doi.org/10.1029/2011RG000373, 2012.

Weiß, M. and Menzel, L.: A global comparison of four potential evapotranspiration equations and their relevance to stream flow modelling in semi-arid environments, Adv. Geosci., 18, 15-23, https://doi.org/10.5194/adgeo-18-15-2008, 2008.

Wild, M., Folini, D., Schär, C., Loeb, N., Dutton, E. G., and KönigLanglo, G.: The global energy balance from a surface perspective, Clim. Dynam., 40, 3107-3134, 2013.
Willett, K. M., Dunn, R. J. H., Thorne, P. W., Bell, S., de Podesta, M., Parker, D. E., Jones, P. D., and Williams Jr., C. N.: HadISDH land surface multi-variable humidity and temperature record for climate monitoring, Clim. Past, 10, 1983-2006, https://doi.org/10.5194/cp-10-1983-2014, 2014.

Willmot, C. J.: On the validation of models, Phys. Geogr., 2, 184194, 1981.

$\mathrm{Xu}, \mathrm{C} .-\mathrm{Y}$. and Singh V. P.: Cross comparison of empirical equations for calculating potential evapotranspiration with data from Switzerland, Water Resour. Manage., 16, 197-219, 2002.

Xu, J., Peng, S., Ding, J., Wei, Q., and Yu, Y.: Evaluation and calibration of simple methods for daily reference evapotranspiration estimation in humid East China, Arch. Agron. Soil Sci., 59, 845858, 2013.

Zambrano-Bigiarini, M.: Hydrogof: Goodness-of-fit functions for comparison of simulated and observed hydrological time series, R package, version 0.3-8, available at: https://cran.r-project.org/ web/packages/hydroGOF/hydroGOF.pdf, last access: 15 December 2015.

Zomer, R. J., Trabucco, A., Bossio, D. A., van Straaten, O., and Verchot, L. V.: Climate change mitigation: A spatial analysis of global land suitability for clean development mechanism afforestation and reforestation, Agr. Ecosyst. Environ., 126, 67-80, 2008. 